\title{
A review on plant growth promoting rhizobacteria acting as bioinoculants and their biological approach towards the production of sustainable agriculture
}

\author{
Vibha Nehra* and Madhu Choudhary ${ }^{1}$ \\ *Department of Microbiology, Kurukshetra University, Kurukshetra-136 119 (Haryana), INDIA \\ ${ }^{1}$ Division of Soil and Crop Management, Central Soil Salinity Research Institute, Karnal-132 001 (Haryana), \\ INDIA \\ *Corresponding author. E-mail: nehra_14@yahoo.in
}

Received: April 13, 2014; Revised received: January 18, 2015; Accepted: April 3, 2015

\begin{abstract}
Plant growth promoting rhizobacteria are the soil bacteria inhabiting around/on the root surface and are directly or indirectly involved in promoting plant growth and development via production and secretion of various regulatory chemicals in the vicinity of rhizosphere. There has been much research interest in PGPB and there is now an increasing number of PGPB being commercialized for various crops. Today a lot of efforts have been made for searching and investigating the PGPB and their mode of action, so that they can be exploited commercially as biofertilizers. Because of the various challenges faced in screening, formulation, and application, PGPB have yet to fulfill their promise and potential as commercial inoculants. Recent progress in our understanding of their diversity, colonization ability, mechanisms of action, formulation, and application should facilitate their development as reliable components in the management of sustainable agricultural systems. Several reviews have discussed specific aspects of PGPB as bioinoculants. We have tried to critically evaluate the current status of bacterial inoculants for contemporary agriculture in developed and developing countries. This review focuses on some important information regarding the biofertilizing potential of some important group of microbes, their formulations, their application for the development of sustainable technology, scope of improvement by genetic engineering, steps to be undertaken for their commercialization and their future prospects.
\end{abstract}

Keywords: Beneficial bacteria, Bioinoculants, PGPB, Carrier, Formulation, Sustainable agriculture

\section{INTRODUCTION}

Different bacterial genera are involved in various biotic activities of the soil ecosystem making it dynamic for nutrient turn over and sustainable for crop production (Ahemad and Khan, 2010a). They stimulate plant growth through mobilizing nutrients in soils, producing numerous plant growth regulators, protecting plants from phytopathogens by controlling or inhibiting them, improving soil structure and bioremediating the polluted soils by sequestering toxic heavy metal species and degrading xenobiotic compounds (like pesticides) (Ahemad and Malik, 2011; Ahemad, 2012). Biofertilizer are defined as the preparation that contains live or latent cells of efficient strains of nitrogen fixing, phosphate solublising or cellulolytic microorganisms. On their application on seeds, soil or composting areas. the number of beneficial microorganisms increases and also enhance the rate of those microbial processes which augment the availability of nutrients that can be easily assimilated by plants. They are also called as the 'microbial inoculants'. Inoculant is the means to transport living bacteria from the factory and introduce them onto living plants, so they may produce the desired effects on plant growth (Tittabutr et al., 2007) which includes nitrogen fixation in legumes, biocontrol of soil-borne diseases, the enhancement of mineral uptake, weathering of soil minerals, and nutritional or hormonal effects. They also help in stimulating the plant growth hormones providing better nutrient uptake and increased tolerance towards drought and moisture stress. They don't have any ill effect on soil health and environment. A small dose of biofertilizer is sufficient to produce desirable results because each gram of carrier of biofertilizers contains at least 10 million viable cells of a specific strain (Anandaraj and Delapierre, 2010).

The most established use of bacterial inoculants is the practice of inoculating legumes with cultures of Rhizobium spp. During late 1970s, Pseudomonas fluorescens and $P$. putida groups began to be intensively investigated (Glick, 1995; Glick and Bashan, 1997) and Azospirillum was found to enhance the growth in the nonlegume plants (Döbereiner and Day, 1976), by directly affecting plant metabolism. In recent, years, evaluation of various other bacterial genera, such as Bacillus, Flavobacterium, Acetobacter, and several Azospirillum- related microorganisms has been done (Tang and Yang, 1997).

In the near history the first commercial preparation of PGPB entered the market but it has been a century that Rhizobium inoculants have been in the market place (Fages, 1992; Tang and Yang, 1997). Nobbe and Hiltner 
launched 'Nitragin' in 1896 using a laboratory culture of Rhizobia, from there the commercialization of biofertilizers started followed by the discovery of Azotobacter and then the blue green algae and a host of other micro-organisms. Azospirillum and Vesicular - Arbuscular Micorrhizae (VAM) are fairly recent discoveries. In India the first study on legume Rhizobium symbiosis was conducted by Joshi (1920) and the first commercial production started as early as 1956 . However, the Ministry of Agriculture under the Ninth Plan initiated the real effort to popularize and promote the input with the setting up of the National Project on Development and Use of Biofertilizers (NPDB).

\section{ORGANISMS ACTING AS BIOINOCULANTS}

The term 'rhizobacteria' implies a group of rhizosphere bacteria competent in colonizing the root environment (Kloepper et al., 1991). Therefore rhizosphere can be defined as any volume of soil specifically influenced by plant roots and/or in association with roots hairs and plant-produced materials (Ahemad and Kibret, 2014). In accordance with Vessey (2003), soil bacterial species burgeoning in plant rhizosphere which grow in, on, or around plant tissues stimulate plant growth by a plethora of mechanisms are collectively known as plant growth promoting rhizobacteria (PGPR). The rhizobacteria are the dominant deriving forces in recycling the soil nutrients and consequently, they are crucial for soil fertility (Glick, 2012) they are commonly used as inoculants for improving the growth and yield of agricultural crops (Ghevariya and Desai, 2014). A number of bacterial species belonging to various genera are associated with the plant rhizosphere and are able to exert a beneficial effect on plant growth (Kumar et al., 2012). Nitrogen (N) is the most vital nutrient for plant growth and productivity. The atmospheric $\mathrm{N}_{2}$ is converted into plant-utilizable forms by biological $\mathrm{N}_{2}$ fixation (BNF) which changes nitrogen to ammonia by nitrogen fixing microorganisms (Biswas and Gresshoff, 2014). Nitrogen fixing organisms are generally categorized as (a) symbiotic $\mathrm{N}_{2}$ fixing bacteria including members of the family rhizobiaceae which forms symbiosis with leguminous plants (e.g. rhizobia) (Ahemad and Khan, 2012) and non-leguminous trees (e.g. Frankia) and (b) non-symbiotic (free living, associative and endophytes) nitrogen fixing forms such as cyanobacteria (Anabaena, Nostoc), Azospirillum, Azotobacter, Gluconoacetobacter diazotrophicus and Azocarus etc. (Bhattacharyya and Jha, 2012). Rhizobia (including Rhizobium, Bradyrhizobium, Mesorhizobium, Sinorhizobium) are generally regarded as microbial symbiotic partners of legumes and are mainly known for their role in the formation of nitrogen -fixing nodules (Antoun and Pre' vost, 2005). However, non-symbiotic nitrogen fixing bacteria provide only a small amount of the fixed nitrogen that the bacterially -associated host plant requires (Glick, 2012). There are many legumes like sesbania which have a very high capacity to fix atmospheric nitrogen and support the growth of the plant (Ladha and Reddy, 1995). Agrobacterium which is a rhizobia related bacteria has also been found to act as a biofertilizer and help in the plant growth (Mia and Shamsuddin, 2010). Phosphate solubilizing microorganisms (PSM), are those micro-organisms that provide the available forms of $\mathrm{P}$ to the plants and hence are the viable substitute to chemical phosphatic fertilizers (Khan et al., 2009). Bacterial genera like Azotobacter, Bacillus, Beijerinckia, Burkholderia, Enterobacter, Erwinia, Flavobacterium, Microbacterium, Pseudomonas, Rhizobium and Serratia are reported as the most significant phosphate solubilizing bacteria (Saharan and Nehra, 2011; Bhattacharyya and Jha, 2012). Vesicular Arbuscular Mycorrhiza (VAM) fungi enhances the availability of phosphorus and nitrogen to host plants and help the plant to survive under a variety of salinity stress conditions (Bargali, 2011).

The PGPR belonging to various bacterial genera are known to participate in many important biological activities (Table 1), such as the biological control of plant pathogens, nutrient cycling and seedling/plant growth (Zahir et al., 2004; Ahemad and Khan, 2010b) through the production of various substances. Among PGPR, Pseudomonas and Bacillus are the most commonly described genera possessing plant growth promoting activities but many other taxa are also included in PGPR group. Selected strains of PGPR are being used as seed inoculant (Sahin et al., 2004; Zahir et al., 2004; Rani et al., 2009; Ahemad and Khan, 2009; Ahemad and Khan, 2010c).

The use of PGPR to augment crop productivity has been limited largely due to the variability and inconsistency of results observed under laboratory, greenhouse and field trials. Soil is an unpredictable environment and an intended result is sometimes difficult to achieve. Climatic variations has also a large impact on the effectiveness of PGPR but sometimes unfavorable growth conditions in the field are to be expected as a normal functioning of agriculture (Zaidi et al., 2009). Despite all these factors, increase in crop yields following PGPR applications in the growth chambers and field trials have also been observed. Plant growth promoting traits do not work independently of each other but additively as it was suggested in the "'additive hypothesis," that multiple mechanisms, such as phosphate solubilization, dinitrogen fixation, ACC deaminase and antifungal activity, IAA and siderophore biosynthesis etc. are responsible for the plant growth promotion and increased yield (Bashan and Holguin, 1997).

\section{FORMULATIONS OF INOCULANT}

In inoculant industry producing a formulation containing an effective bacterial strain is a crucial aspect and can determine the success or failure of a biological agent (Bashan, 1998). A microorganism which is functioning optimally under laboratory conditions might not be 
able to produce equivalent results under field conditions after formulation production. Once an inoculant formulation which works in situ has been developed, it must be refined to allow for the sophistication of the end-user (Stephens and Rask, 2000). It is imperative that the formulation remain stable during production, distribution, storage, and transportation, irrespective of whether product is new or improved. The formulation produced should also be easy to handle and apply by the end users, it should be delivered to the target site in the most appropriate manner and form, it should be able to protect the agent from various harmful environmental factors, and should be able to maintain or enhance activity of the organism in the field (Jones and Burges, 1998). Another important consideration is the cost-effectiveness of the formulation it should not put much pressure on the end users financially (Xavier et al., 2004).

Optimal characteristics of a carrier for inoculants: The delivery vehicle of live microorganisms from the factory to the field is called carrier (Trevors et al., 1992). The carrier is the major portion (by volume or weight) of the inoculant that helps to deliver a suitable amount of PGPM in good physiological condition (Smith, 1992). The carrier should be designed to provide a suitable microenvironment for the PGPM and should assure a sufficient shelf life of the product (at least 2-3 months for commercial purposes, possibly at room temperature). The formulation should allow an easy dispersion or dissolution in the volume of soil near the root system. A good carrier should therefore posses as much as the following properties: good moisture absorption capacity, easy to process and free of lump-forming materials, near-sterile or easy to sterilize by autoclaving or by other methods (e.g., gamma-irradiation), low cost and availability in adequate amounts, and good $\mathrm{pH}$ buffering capacity (Keyser et al., 1993). For carriers that shall be used for seed coating, a good adhesion to seeds is also important. Other characteristics that are affecting the carrier appropriateness are a standardized composition ensuring chemical and physical stability, suitability for as many PGPM species and strains as possible, the possibility of mixing with other compounds (i.e., nutrients or adjuvants), and being composed of biodegradable and nonpolluting compounds (Smith, 1992). In case the inoculant is used as seed coating, the carrier shall assure the survival of the PGPM on the seed since normally seeds are not immediately sown after seed coating (Muresu et al., 2003). A good carrier should have one essential characteristic: the capacity to deliver the right number of viable cells in good physiological condition at the right time (Smith, 1992; Trevors et al., 1992).

\section{CARRIERS}

Various organic carriers and inorganic substances have been used as carriers. The organic carriers suffer from the disadvantage that due to their high nutrient content, they support the growth of a large number of contaminants as well. Also, during heat sterilization of the formulations, organic carriers may release some compounds, which are toxic to bacteria resulting in low bacterial counts (Weiss et al., 1987). While selecting the inorganic carriers, we consider their bulk density and local availability. Any carrier-based formulation with low bulk density, such as vermiculite, will require larger packing volume per kilogram of the formulation (Saharan et al., 2010).

Talc formulation: Talc, chemically referred as magnesium silicate $\left(\mathrm{Mg}_{3} \mathrm{Si}_{4} \mathrm{O}_{10}(\mathrm{OH})_{2}\right.$, is used as a carrier for formulation development due to its inert nature and easy availability as raw material from soapstone industries. The potential of talc to be used as a carrier was demonstrated by Kloepper and Schroth (1981). Rhizobacteria could survive in talc for 2 months. The Fluorescent Pseudomonads after storage for two months in talc mixture with $20 \%$ xanthum gum at $4^{\circ} \mathrm{C}$ did not decline in no. While $P$. fluorescens isolate Pf1could survived up to 240 days in storage (Vidhyasekaran and Muthamilan, 1995). P. putida strain 30 and 180 survived up to 6 months (Bora et al., 2004) and B. Subtilis survived up to 45 days in talc based formulations (Amer and Utkhede, 2000). Saravanakumar et al. (2007a) demonstrated that application of talc-based bioformulation of $P$. fluorescens Pf1 consistently reduced the blister blight disease and increased the yield on tea plants. The same research group has demonstrated that $P$. fluorescens Pf1 effectively controlled the dry root rot disease on mung bean plants (Saravanakumar et al., 2007b). Further, seed treatment, soil application and seedling dip of talc-based bioformulation of Pf1 effectively reduced the sheath rot disease on rice plants under glasshouse and field conditions (Manikandan et al., 2010).

Press mud formulation: Press mud is a by-product of sugar industries. Its usefulness as fertilizer is based on the nutrient content of the mud. It is rich in micronutrients and can reduce the requirement of chemical fertilizers. It also provides suitable conditions to bacteria for carrying out nitrogen fixation and phosphate solubilisation that keeps the soil healthy and develops the self-reclamation cycle. The fertilizer produced is free from all pathogens, harmful bacteria, weeds and seeds due to the high temperature produced during biocomposting. Fertilizer is easy to handle, to pack and transport. The biocompost contains $25-30 \%$ organic carbon, $1.2-2.0 \%$ nitrogen, $1.5-2.0 \%$ phosphorous and $2.5-3.0 \%$ potash (Partha and Sivasubramanian, 2006). This carrier maximizes the survival of Azospirillum spp. by providing favourable conditions in comparison to lignite, which is predominantly used as a carrier material in India (Muthukumarasamy et al., 1999).

Vermiculite formulation: Vermiculite is a naturally occurring layer silicate mineral $\left[\left(\mathrm{Si}_{3} \mathrm{Al}\right) \mathrm{Mg}_{3}(\mathrm{OH})\right.$ $\left.{ }_{2} \mathrm{O}_{10} \cdot \mathrm{Mg}_{0.5} \cdot \mathrm{nH}_{2} \mathrm{O}\right]$ (Alexandre-Franco et al., 2011; Bozzolo and Evans, 2013) and could also be considered as possible carriers, especially when the process of 
their production involves the use of specific selected strains. For example, increased amount of $\mathrm{N}$ and $\mathrm{P}$ availability in the final product can be achieved by adding $\mathrm{N}$-fixing and P-solubilizing bacteria to a vermicompost (Vassileva et al., 2010). It can be sterilized easily due to its inorganic and pre expanded nature by the common sterilization processes without the risk of producing toxic by-products or causing further structural changes. It provides enough space for microbial proliferation and also provides superior aeration due to its multilamellate structure. It is widely available and is relatively less expensive (Meisinger, 1984). So vermiculite has become a very attractive material for the inoculant production due to the various properties exhibited by it like anticrusting (Hemphill Jr., 1982), moisture-holding, and plant growth promoting (Lima et al., 1984; Reid et al., 1983) abilities. Vidhyasekaran and Muthamilan (1995) reported P. fluorescens (Pf1) to survive for 8 months and B. Subtilis for 45 days (Amer and Utkhede, 2000) in vermiculite formulation.

Peat formulations: Peat formulations have been the carriers of choice, and are the most commonly used in the rhizobia inoculation industry (Kaljeet et al., 2011). Peat is widely available and has a long history of field trials, therefore commonly used as a carrier for PGPR, particularly for rhizobia inoculants. Peat inoculant applied to the seed as slurry is the most commonly -used method to inoculate grain legumes with rhizobia (e.g. Bradyrhizobium spp., Mesorhizobium spp., Rhizobium spp. etc.). Peat slurry inoculants are made using finely -milled peat that have been sterilised by gamma irradiation and these sterilised inoculants can support high concentrations of rhizobia, generally $10^{9}$ to $10^{10}$ cellsg $^{-1}$ peat at manufacture (Hartley et al., 2005). Further, the use of a sterile peat carrier significantly reduces threats to quality resulting from the presence of contaminants. Sterile peat also lowers costs of culture production by extending the broth, through culture dilution, while still achieving a higher final population density (Stephens and Rask, 2000). Further to ensure contact between the rhizobia and the legume seed coat, and to reduce rhizobial desiccation inoculation is improved by the use of adhesives and polymers (Deaker et al., 2004). The peat inoculant is not difficult to produce, is easy to apply and usually maintains a high concentration of viable bacteria. In this form, the bacteria are metabolically active, and bacterial multiplication continues during the storage period also as long as sufficient nutrients, moisture, and the correct temperature are maintained in some inoculants. Since peat was adopted decades ago, farmers are by now quite comfortable using it, and governmental agencies are also very familiar with how to monitor its quality.

The principle drawbacks originate from the different batches of peat and peat collected from various sources differ greatly in composition, structure, $\mathrm{pH}$ and microbial populations (Graham-Weiss et al., 1987). Due to this the final product is greatly affected and may cause difficulties in deciding the inoculant dosage, storage conditions (Van Elsas and Heijnen, 1990), and inoculant variation in effectiveness between different manufacturers and between different batches from the same manufacturer (Bashan et al., 1992). Some peat has been known to contain inhibitors to Rhizobium strains (Brockwell, 1985). Due to organic nature of peat, it cannot be completely sterilized by steam or by gamma irradiation because toxic by-products are produced due to high temperatures and high dosage of irradiation (Mulligen and Cooper, 1985) and it also undergoes structural and compositional changes which are unfavourable for subsequent growth and survival of the bacterial spp. (Strijdom and van Rensburg, 1981). From the delivery standpoint, peat powder is easily blown away from the seeds by the commonly used seed air-delivery system used by the planter. Peat interferes with the seed monitoring mechanism of the planters which has been rectified by the addition of adhesives to peat formulations, which has also ensured enhanced seed coverage.

Other alternatives of peat: Due to increase in demand and cost rise of peat as a substrate in horticulture have led to the search of an alternative substrate which possess high quality and low cost (Gil et al., 2008; Moral et al., 2009). A number of studies have shown that organic residues such as urban solid wastes, sewage sludge, animal manure and dung, paper waste, pruning waste, spent mushroom and even green wastes, after proper composting, can be used with very good results as container growth substrates instead of peat (Bustamante et al., 2008; Moral et al., 2009). Cattle manure compost (CMC) (Ko et al., 2008), freeze-dried cells (McInnes and Date, 1999) and lyophilized rhizobial cells (Caesar and Burr, 1991) can also be used as an alternatives to peat.

All of the above mentioned carriers rely on the absorption of the microorganisms by the substance/matrix of the carrier but this method has some drawbacks, particularly in context with the survival of the microorganisms and their protection during transport, storage, and handling. Nevertheless, some processes with different carriers using such approach have been patented: the patent no. 521.850 of Belgian for Rhizobium which uses diatomaceous earth and colloidal silica; the British patent no. 1.777.077 for the use of bentonite for Rhizobium; French Patent no. 1.180.000 for the Azotobacter group using a must juice, to which substances such as cellulose, bone meal, kaolin, or silica gel are added which has an adsorbing action; United States Patent no. 4956295 for the stabilization of dried bacteria extended in particulate carriers, where dried viable bacteria are mixed in a particulate carrier composed primarily of an inorganic salt such as sodium or calcium carbonates, bicarbonates, sulfates, or phosphates of low moisture absorbing capacity together with a minor proportion of a silica gel absorbent. Different types of carriers used for inoculant development are mentioned in table 2. 
Table 1. Beneficial Interactions between plant growth promoting rhizobacteria.

\begin{tabular}{|c|c|c|c|c|}
\hline PGPR & Hosts & Colonization & $\begin{array}{c}\text { Plants } \\
\text { Response }\end{array}$ & Reference(s) \\
\hline $\begin{array}{l}\text { Rhizobium } \\
\text { meliloti } \\
\text { Pseudomonas }\end{array}$ & $\begin{array}{l}\text { Medicago sativa, Medicago } \\
\text { olymorph, Melilotus sp., } \\
\text { rigonella foenum-graecum } \\
\text { rigonella sp., Trifolium spp }\end{array}$ & Roots & $\begin{array}{l}\text { Biological nitrogen } \\
\text { fixation }\end{array}$ & $\begin{array}{l}\text { (Maheshwari } \\
\text { et al., } 2010 \\
\text { Zhao et al., 2012) }\end{array}$ \\
\hline $\begin{array}{ll}\text { Pseudomonas } & \text { Pis } \\
\text { Pseudomonas sp } & \text { Lat } \\
\text { Strain 267 and R., } & \text { fab } \\
\text { Azopirillum lipoferum } & \text { lent } \\
\text { Trifolium spp } & \end{array}$ & $\begin{array}{l}\text { Pisum spp., } \\
\text { athyrus spp. Vicia } \\
\text { aba, Vicia } \\
\text { entils, Lens spp., }\end{array}$ & Roots & $\mathrm{BNF}$ & $\begin{array}{l}\text { (Tchebotar } \\
\text { et al., 1998; } \\
\text { (Mishra et al., } \\
\text { 2011) }\end{array}$ \\
\hline $\begin{array}{l}\text { Pseudomonas sp } \\
\text { and Bacillus } \mathrm{sp} \\
\text { Rhizobium Phaseoli }\end{array}$ & $\begin{array}{l}\text { haseolus vulgaris, } \\
\text { haseolus coccineus, } \\
\text { icia faba }\end{array}$ & Roots & $\begin{array}{l}\text { Increases nodulation } \\
\text { in bean }\end{array}$ & $\begin{array}{l}\text { (Stajkovic et al., } \\
\text { 2011) }\end{array}$ \\
\hline $\begin{array}{l}\text { Rhizobium } \\
\text { A. brasilene } \\
\text { Pseudomonas, } \\
\text { B. megaterium }\end{array}$ & $\begin{array}{l}\text { icer arietinum, Dactylis } \\
\text { lomerata, Phaseolus } \\
\text { ulgaris }\end{array}$ & Roots & $\begin{array}{l}\text { BNF and Phosphate } \\
\text { solubilization }\end{array}$ & $\begin{array}{l}\text { (Gunasekaran } \\
\text { et al., 2004; } \\
\text { (Dardanelli et } \\
\text { al., 2008) }\end{array}$ \\
\hline $\begin{array}{l}\text { Bradyrhizobium } \\
\text { sp., Bacillus sp., } \\
\text { Serratia } \\
\text { marcescens }\end{array}$ & $\begin{array}{l}\text { igna radiata, } \\
\text { rachis hypogaea, } \\
\text { lycine max }\end{array}$ & Roots & $\mathrm{BNF}$ & $\begin{array}{l}\text { (Badawi et al., } \\
\text { 2011; Black et } \\
\text { al., 2012) }\end{array}$ \\
\hline $\begin{array}{l}\text { B. japonicum } \\
\text { Azospirillum brasilense, } Z \\
\text { Pseudomonas, Bacillus } \\
\text { subtilis, B. thuringiensis } \\
\text { Aeromonas sp, Serratia sp }\end{array}$ & $\begin{array}{l}\text { Glycine max, } \\
\text { Zea mays L. }\end{array}$ & Rhizosphere & $\begin{array}{l}\text { BNF, promote } \\
\text { seed germination } \\
\text { and early seedling } \\
\text { growth }\end{array}$ & (Bai et al., 2003) \\
\hline $\begin{array}{lr}\text { AM fungi } & P \\
\text { free living } \mathrm{N}_{2} & S o \\
\text { fixing bacteria like } & L \\
\text { Azospirillum brasilense } & T r \\
\text { or Azotobacter } & \text { a } \\
\text { Pseudomomas sp } & \end{array}$ & $\begin{array}{l}\text { Pinus sabiniana, } \\
\text { olanum lycopersicum, } \\
\text { Lactuca sativa, } \\
\text { Triticum aestivum } \\
\text { and Zea mays }\end{array}$ & Roots & $\begin{array}{l}\text { stimulates root colonization, } \\
\text { BNF, Increases biomass, } \\
\text { limits soil salinity stress, } \\
\text { and affects plant yield }\end{array}$ & $\begin{array}{l}\text { (Kohler } \\
\text { et al., 2010) }\end{array}$ \\
\hline $\begin{array}{l}\text { Pseudomonas } \\
\text { Bacillus } \\
\text { Mycobacterium }\end{array}$ & Zea mays & Roots & $\begin{array}{l}\text { stimulates plant growth, } \mathrm{N} \text {, } \\
\mathrm{P} \& \mathrm{~K} \text { uptake in nutrient def- } \\
\text { cient soil }\end{array}$ & $\begin{array}{l}\text { (Egamberdiyeva } \\
\text { 2007) }\end{array}$ \\
\hline $\begin{array}{l}\text { Bacillus substilis strain } 101 \\
\text { Azospirillum Brasilemse } \\
\text { sp. } 245\end{array}$ & 1 Lycopersicon esculentum & Rhizosphere & $\begin{array}{l}\text { coinoculation shows more } \\
\text { Plant height,node number } \\
\text { total biomass }\end{array}$ & $\begin{array}{l}\text { (Felici } \\
\quad \text { et al., 2008) }\end{array}$ \\
\hline $\begin{array}{l}\text { Pseudomonas putida.Azo } \\
\text {-spirilium, Azotobacter }\end{array}$ & $\begin{array}{l}\text { Artichoke (cynara } \\
\text { Scolymus) }\end{array}$ & Rhizosphere & $\begin{array}{l}\text { Phosphate solublizing bacteria along } \\
\text { with nitrogen fixing bacteria led to } \\
\text { significant increase in radicle and } \\
\text { shoot length, shoot weight, coefficient } \\
\text { of velocity of germination, seedling } \\
\text { vigority index, and significant decrease } \\
\text { in mean time of germination }\end{array}$ & $\begin{array}{c}\text { (Jahanian } \\
\text { et al., 2012) }\end{array}$ \\
\hline Pseudomonas sp.A3R3 & $\begin{array}{l}\text { Allysum serpyllifolium } \\
\text { Brassica juncea }\end{array}$ & Rhizosphere & $\begin{array}{l}\text { Increased significantly the biomass } \\
\text { (B. juncea) and Ni content (A. } \\
\text { serpyllifolium) in plants grown in } \\
\text { Ni-stressed soil }\end{array}$ & $\begin{array}{l}\text { (Ma et al., } \\
\text { 2011) }\end{array}$ \\
\hline $\begin{array}{l}\text { Pseudomonas } \text { sp. SRI2 } \\
\text { Psychrobacter } \mathrm{sp} \text {. SRS8 } \\
\text { Bacillus } \text { sp. SN9 }\end{array}$ & $\begin{array}{l}\text { Brassica juncea, } \\
\text { Brassica oxyrrhina }\end{array}$ & Rhizosphere & $\begin{array}{l}\text { Increased the biomass of the test } \\
\text { plants and enhanced } \mathrm{Ni} \text { accumulation } \\
\text { in plant tissues }\end{array}$ & $\begin{array}{r}\text { ( Ma et al., } \\
\text { 2009a) }\end{array}$ \\
\hline $\begin{array}{l}\text { Psychrobacter } \text { sp.SRA1 } \\
\text { Bacillus cereus SRA10 }\end{array}$ & $\begin{array}{l}\text { Brassica juncea, } \\
\text { Brassica oxyrrhina }\end{array}$ & Rhizosphere & $\begin{array}{l}\text { Enhance the metal accumulation in } \\
\text { plant tissue by facilitating the release } \\
\text { of Ni from non soluble phase in } \\
\text { the soil. }\end{array}$ & $\begin{array}{l}\text { ( Ma et al., } \\
\text { 2009b) }\end{array}$ \\
\hline $\begin{array}{l}\text { Pseudomonas aeruginosa } \\
\text { Pseudomonas fluorescens } \\
\text { Ralstonia metallidurans }\end{array}$ & Maize & Rhizosphere & $\begin{array}{l}\text { Promoted plant growth, facilitated } \\
\text { soil metal mobilization, enhanced } \\
\mathrm{Cr} \text { and } \mathrm{Pb} \text { uptake }\end{array}$ & $\begin{array}{l}\text { (Braud et al., } \\
\text { 2009) }\end{array}$ \\
\hline
\end{tabular}




\begin{tabular}{|c|c|c|c|c|}
\hline $\begin{array}{l}\text { Bradyhizobium sp. } 750 \\
\text { Pseudomonas sp., } \\
\text { Ochrobactrum cytisi }\end{array}$ & Lupinus luteus & Rhizosphere & $\begin{array}{l}\text { Increased both biomass and } \\
\text { nitrogen content,accumulation } \\
\text { of metals (phytostabilisation } \\
\text { potential) }\end{array}$ & $\begin{array}{l}\text { (Dary et al., } \\
\text { 2010) }\end{array}$ \\
\hline $\begin{array}{l}\text { Pseudomonas putida } \\
\text { CC-R2-4, Bacillus } \\
\text { subtilis CC-pg } 104\end{array}$ & Lectuca sativa $\mathrm{L}$. & Rhizosphere & $\begin{array}{l}\text { Significant increase in shoot } \\
\text { length and root length achieved } \\
\text { through encapsulated inoculant }\end{array}$ & $\begin{array}{l}\text { (Rekha et al., } \\
\text { 2007) }\end{array}$ \\
\hline $\begin{array}{l}\text { Pseudomnas putida strain } \\
\text { R-168, Pseudomonas fluores } \\
\text { cens strain R-93,Pseudomonas } \\
\text { fluorescens DSM 50090, } \\
\text { Pseudomonas putida DSM291 } \\
\text { Azospirillum lipoferum DSM } \\
\text { 1691,Azospirillum brasilense } \\
\text { DSM } 1690\end{array}$ & $\begin{array}{l}\text { Maize (Zea mays L.) } \\
1 \\
1 \\
\\
\end{array}$ & Rhizosphere & $\begin{array}{l}\text { Plant height, seed weight.no. } \\
\text { of seeds per ear and leaf area, } \\
\text { shoot dry weight significantly } \\
\text { increased. }\end{array}$ & $\begin{array}{l}\text { (Gholami et } \\
\text { 2009) }\end{array}$ \\
\hline $\begin{array}{l}\text { Bacillus subtilis, } \\
\text { Pseudomonas aeruginosa }\end{array}$ & $\begin{array}{l}\text { Solanum lycopersicum } \\
\text { L(.tomato),Abelmoschus } \\
\text { esculentus(okra)Amar- } \\
\text { anthus } p \text {.(African spinach }\end{array}$ & Rhizosphere & $\begin{array}{l}\text { Dry biomass increased } 31 \% \\
\text { for tomato, } 36 \% \text { for okra } \\
83 \% \text { for African spinach }\end{array}$ & $\begin{array}{l}\text { (Adesemoye } \\
\text { et al., 2008) }\end{array}$ \\
\hline \multicolumn{2}{|c|}{$\begin{array}{l}\text { Pseudomonas tolaasii ACC23, Brassica napus } \\
\text { Pseudomonas fluorescens ACC9, } \\
\text { Alcaligenes } \mathrm{sp} \text {. ZN4, } \\
\text { Mycobacterium } \mathrm{sp} \text {. ACC } 14\end{array}$} & Rhizosphere & $\begin{array}{l}\text { Protected Canola plants } \\
\text { against the inhibitory effects } \\
\text { of Cadmium }\end{array}$ & $\begin{array}{l}\text { (Dell' Amico } \\
\text { et al., 2008) }\end{array}$ \\
\hline $\begin{array}{l}\text { Azotobacter chroococum } \\
\text { Azospirillum lipoferum }\end{array}$ & $\begin{array}{l}\text { Cotton (Gossypium } \\
\text { hirsutum L.) }\end{array}$ & Rhizosphere & $\begin{array}{l}\text { Seed yield ( } 21 \%) \text {, plant } \\
\text { height }(5 \%) \text { and microbial } \\
\text { population in soil }(41 \%) \\
\text { increased over their respective } \\
\text { controls while boll weight and } \\
\text { staple length remained statistically } \\
\text { unaffected }\end{array}$ & $\begin{array}{l}\text { (Anjum et al., } \\
\text { 2007) }\end{array}$ \\
\hline $\begin{array}{l}\text { Bacillus subtilis, } \\
\text { Bacillus pumis, } \\
\text { Rhizobium sp. IC3 } 123\end{array}$ & Cajanus cajan & Rhizosphere & $\begin{array}{l}\text { Increase in plant fresh weight, } \\
\text { chlorophyll content, nodule. }\end{array}$ & $\begin{array}{l}\text { (Rajendran } \\
\text { et al., } \\
\text { 2008) }\end{array}$ \\
\hline $\begin{array}{l}\text { Bacillus sp. Paeni- } \\
\text { bacillus } \mathrm{sp} .\end{array}$ & Rice & Roots & $\begin{array}{l}\text { Promote significantly root } \\
\text { and shoot growth }\end{array}$ & $\begin{array}{l}\text { (Beneduzi et al., } \\
\text { 2008) }\end{array}$ \\
\hline $\begin{array}{l}\text { Bacillus cereus } \mathrm{RS} 18 \\
\text { Bacillus licheniformis } \mathrm{RC} 08\end{array}$ & $\begin{array}{l}\text { Wheat, } \\
\text { Spinach }\end{array}$ & Roots & $\begin{array}{l}\text { All bacterial strains were } \\
\text { Effective in IAA produc- } \\
\text { tion and significantly incresaed } \\
\text { growth of wheat and spinach }\end{array}$ & $\begin{array}{c}\text { (Cakmakci } e t \\
\text { al., 2007) }\end{array}$ \\
\hline $\begin{array}{l}\text { Xanthomonas sp. RJ3, } \\
\text { Azomonas } \text { sp. RJ4 } \\
\text { Pseudomonas sp. RJ10, } \\
\text { Bacillus } \mathrm{RJ} 31\end{array}$ & Brassica napus & Rhizosphere & $\begin{array}{l}\text { Stimulated plant growth and } \\
\text { Increased cadmium } \\
\text { accumulation }\end{array}$ & $\begin{array}{l}\text { (Sheng and } \\
\text { Xia, 2006) }\end{array}$ \\
\hline $\begin{array}{l}\text { Pseudomonas sp., } \\
\text { Bacillus sp. } \\
\text { Ochrobactrum } \\
\text { Bacillus cereus }\end{array}$ & $\begin{array}{l}\text { Mustard } \\
\text { Mungbean }\end{array}$ & $\begin{array}{l}\text { Rhizosphere } \\
\text { Rhizosphere }\end{array}$ & $\begin{array}{l}\text { Stimulated plant growtjh and } \\
\text { Decreased CR (VI) Content } \\
\text { Lower the toxicity of cadmium } \\
\text { to seedlings by reducing } \mathrm{Cr} \text { (VI) } \\
\text { to } \mathrm{Cr} \text { (III) }\end{array}$ & $\begin{array}{l}\text { (Rajkumar et } \\
\text { al., 2006) } \\
\text { (Faisal and } \\
\text { Hasnain, } \\
\text { 2005) }\end{array}$ \\
\hline $\begin{array}{l}\text { Azospirillum brasilense, } \\
\text { Bacillus pantothenticus, } \\
\text { Pseudomonas pieketti }\end{array}$ & $\begin{array}{l}\text { Rice } \\
\text { (Oryza sativa) }\end{array}$ & Rhizosphere & $\begin{array}{l}\text { Increased rice grain yield } \\
\text { maximum upto } 76.9 \%\end{array}$ & $\begin{array}{l}\text { (Thakuria et } \\
\text { al., 2004) }\end{array}$ \\
\hline $\begin{array}{l}\text { Pseudomonas fluorescens } \\
\text { PGPR1, PGPR2, PGPR4 }\end{array}$ & $\begin{array}{l}\text { Peanut } \\
\text { (Archis hypogoea L.) }\end{array}$ & Rhizosphere & $\begin{array}{l}\text { Significantly enhanced pod } \\
\text { yield,haulm yield and nodule } \\
\text { dry weight over the control }\end{array}$ & $\begin{array}{l}\text { (Day et } \\
\text { al., 2004) }\end{array}$ \\
\hline
\end{tabular}

\section{POLYMER- BASED POLYMERS}

Alginate formulations: Alginate is the most commonly used substance for microbial cell encapsulation. It is a natural polymeric compound made up of D -mannuronic acid and L-glucuronic acid. It is derived mainly from brown macroalgae such as Macrocystis pyrifera (kelp), but recently it has been found that another macroalga (Sargassum sinicola) produce alginate of similar physical characteristics (Yabur et al., 2007). It is also available from several bacteria (Pseudomonas and Azotobacter) (Hay et al., 2010). Alginate beads generally have a diameter of $2-3 \mathrm{~mm}$, but microbeads with a size of 50 to $200 \mu \mathrm{m}$ that can entrap up to $10^{8}$ to $10^{9} \mathrm{CFUg}^{-1}$ have also been proposed (Bashan et al., 2002). Different AMF structures have also been entrapped into alginate matrixes 
Table 2. Carriers materials used for biofertilizers.

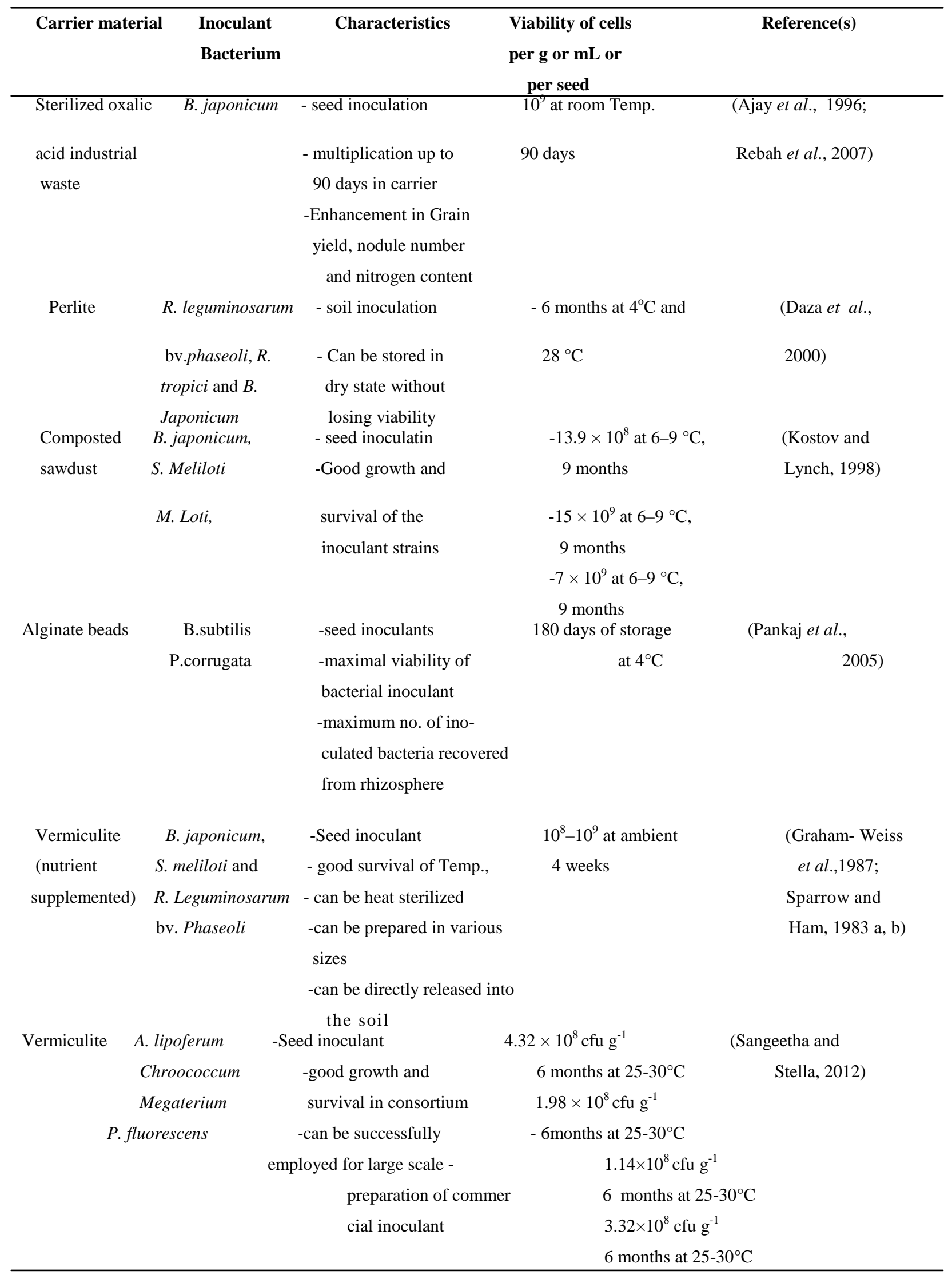




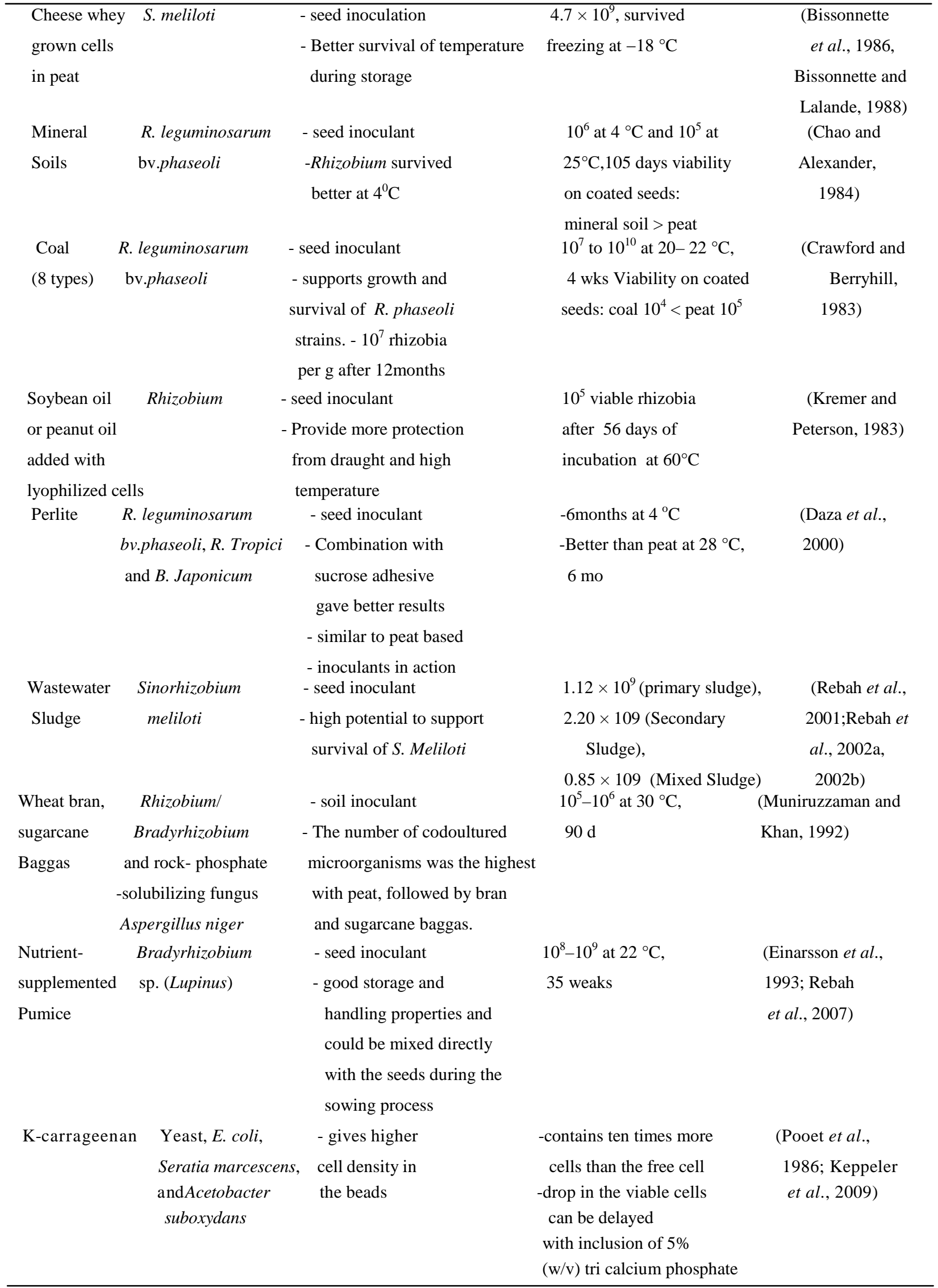




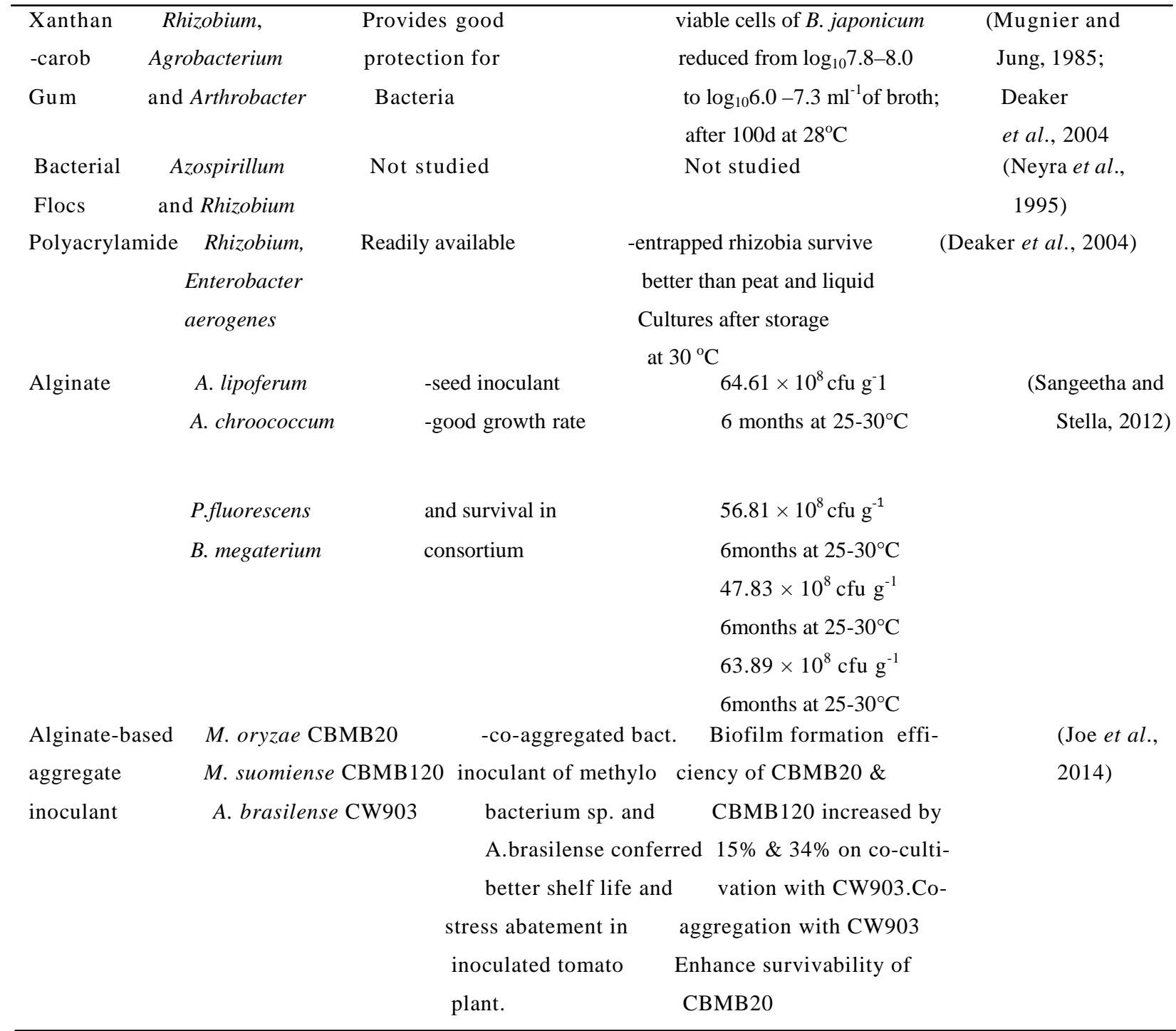

(Strullu and Plenchette, 1991) or in beads formed with different polymers (Vassilev et al., 2005). Encapsulation of living cells in polymeric gel is a well-established technology in a wide range of different applications (Park and Chang, 2000). The gel-like matrix with its catalytic ability allows the cells to remain viable for longer duration. Moreover, alginate beads entraps sufficient number of bacteria (Zohar-Perez et al., 2002) which shows several advantages over free cell formulations like, it protects the bacteria from biotic stresses (Smit et al., 1996) and abiotic stresses such as the inhibitory effect of toxic compounds (Cassidy et al., 1997), enhanced survival and improved physiological activity (Weir et al., 1995), supply of encapsulated nutritional additives (Trevors et al., 1993), increased cell densities and preferential cell growth in various internal aerobic and anaerobic zones of encapsulating gel. This technology was firstly used to encapsulate the plant-beneficial bacteria like $A$. brasilense and $P$. fluorescens (Bashan, 1986), which were later successfully used to inoculate wheat plants under field conditions. The bacteria survived in the field long enough and their populations were comparable to the survival of bacteria originating from peat-based inoculants (Bashan et al., 1987). Inclusion of filamentous fungi such as Aspergillus (Jain et al., 2010) has been also proved possible.

Alginate beads can maintain a sufficient amount of live cells to assure inoculation up to several months (van Veen et al., 1997). The viability of inocula can be improved by adding some nutrients (e.g., skimmed milk) to the inoculum (Hernandez et al., 2006) or freeze-drying gel beads in presence of glycerol (Tal et al., 1997) .However, freeze-drying of alginate beads can result in some collapse of the matrix (Rassis et al., 2002) therefore, while planning this technological process some materials can be added to the mixture which can reduce the cost and/or improve the mechanical properties. Adding chitin to the beads (Zohar-Perez et $a l ., 2005)$ helped preserve their porous cellular structure resulting in significantly higher porosity values when compared to starch filled beads (Tal et al., 1999) and resulted in higher bacterial efficacy when evaluating their effect on plants. Addition of $0.5 \%$ kaolin to freeze 
Table 3: Comparison of vermiculite, peat and alginate related to production of bacterial inoculants.

\begin{tabular}{|c|c|c|c|c|}
\hline Properties & Vermiculite & Peat & Alginate & Reference(s) \\
\hline $\begin{array}{l}\text { Buffering } \\
\text { capacity }\end{array}$ & Approximately neutral & $\begin{array}{l}\text { Usually require } \\
\text { neutralization before } \\
\text { use as carrier } \\
\text { Little }\end{array}$ & $\begin{array}{l}\text { Used at low } \mathrm{pH} \\
\text { for cell immobilization } \\
\text { and encapsulation } \\
\text { Good }\end{array}$ & $\begin{array}{l}\text { (Graham-Weiss et al., } \\
\text { 1987; Pandey } \\
\text { and Khuller, 2005) } \\
\text { (Orlando et al., 1994; } \\
\text { Bashan et al., 2002) }\end{array}$ \\
\hline Toxicity & $\begin{array}{l}\text { Inorganic; will not } \\
\text { produce organic toxic } \\
\text { products or undergo } \\
\text { structural changes } \\
\text { sterilization }\end{array}$ & $\begin{array}{l}\text { Organic; known to } \\
\text { occasionally contain } \\
\text { inhibitors to bacterial } \\
\text { strains; upon may } \\
\text { produce toxic substances } \\
\text { and undergo compositional } \\
\text { and structural changes } \\
\text { upon sterilization }\end{array}$ & $\begin{array}{l}\text { Produces no environment } \\
\text { pollution, non -toxic and } \\
\text { biodegradable }\end{array}$ & $\begin{array}{l}\text { (Bashan, 1998; } \\
\text { Daza et al., } \\
\text { 2000) }\end{array}$ \\
\hline Contamination & $\begin{array}{l}\text { Exfoliated at extremely } \\
\text { high temperature which } \\
\text { kills microorganisms; } \\
\text { its mineral nature does } \\
\text { not support microbial } \\
\text { growth }\end{array}$ & $\begin{array}{l}\text { Usually contains unknown } \\
\text { microbial contaminants } \\
\text { able to grow on } \\
\text { organic compounds }\end{array}$ & $\begin{array}{l}\text { Cannot be contaminated } \\
\text { after Production }\end{array}$ & $\begin{array}{l}\text { (Bashan, 1998; } \\
\text { Young et al., } \\
\text { 2006) }\end{array}$ \\
\hline $\begin{array}{l}\text { Physical } \\
\text { properties }\end{array}$ & $\begin{array}{l}\text { Multilamellate; provides } \\
\text { good aeration, quick } \\
\text { temp equilibration } \\
\text { and space for microbial } \\
\text { growth during fermentation }\end{array}$ & $\begin{array}{l}\text { Not layered; structure may } \\
\text { change at high temperature } \\
\text { or upon exposure to strong } \\
\text { gamma radiations acid } \\
\text { n }\end{array}$ & $\begin{array}{l}\text { Physically uniform, } \\
\text { Composed of f1-1, 4- } \\
\text { linked D-mannuronic } \\
\text { and L-glucuronic acid, } \\
\text { provides good aeration, } \\
\text { mechanical strength and } \\
\text { protects from abiotic } \\
\text { stress }\end{array}$ & $\begin{array}{l}\text { (Klein et al., } \\
\text { 1983; Hartley } \\
\text { et al., 2005) }\end{array}$ \\
\hline $\begin{array}{l}\text { Seed sticking } \\
\text { ability }\end{array}$ & $\begin{array}{l}\text { Flaky; good sticking } \\
\text { properties for seed } \\
\text { coating }\end{array}$ & $\begin{array}{l}\text { Powder or granular; } \\
\text { often requires sticker } \\
\text { to adhere to seeds }\end{array}$ & $\begin{array}{l}\text { In the form of macro } \\
\text { or microbeads; requires } \\
\text { adhesives for the } \\
\text { attachment to the seeds }\end{array}$ & $\begin{array}{l}\text { (Graham-Weiss } \\
\text { et al., 1987; } \\
\text { Bashan et } \\
\text { al., 2002) }\end{array}$ \\
\hline $\begin{array}{l}\text { Industrial } \\
\text { application }\end{array}$ & $\begin{array}{l}\text { Extensively used in } \\
\text { industries for various } \\
\text { purposes }\end{array}$ & $\begin{array}{l}\text { Various successful } \\
\text { industrial processes } \\
\text { exist }\end{array}$ & $\begin{array}{l}\text { No inexpensive } \\
\text { industrial technology } \\
\text { exists }\end{array}$ & $\begin{array}{l}\text { (Alexandre- } \\
\text { Franco et al., } \\
\text { 2011) }\end{array}$ \\
\hline $\begin{array}{l}\text { Consumer } \\
\text { friendly }\end{array}$ & $\begin{array}{l}\text { Requires larger packing } \\
\text { volume per kilogram }\end{array}$ & $\begin{array}{l}\text { Simple to use for } \\
\text { the farmer }\end{array}$ & $\begin{array}{l}\text { Simple to use for } \\
\text { the farmer }\end{array}$ & $\begin{array}{l}\text { (Kalra et al., } \\
\text { 2010; } \underline{\text { Siddiqui }}\end{array}$ \\
\hline & of the formulation so a & & & and Kataoka, \\
\hline & bit clumsy for the farmer & & & 2011) \\
\hline Storage space & Bulk & Bulk & $\begin{array}{l}\text { Storage requires little } \\
\text { space }\end{array}$ & $\begin{array}{l}\text { (Saharan et al., } \\
\text { 2010) }\end{array}$ \\
\hline
\end{tabular}




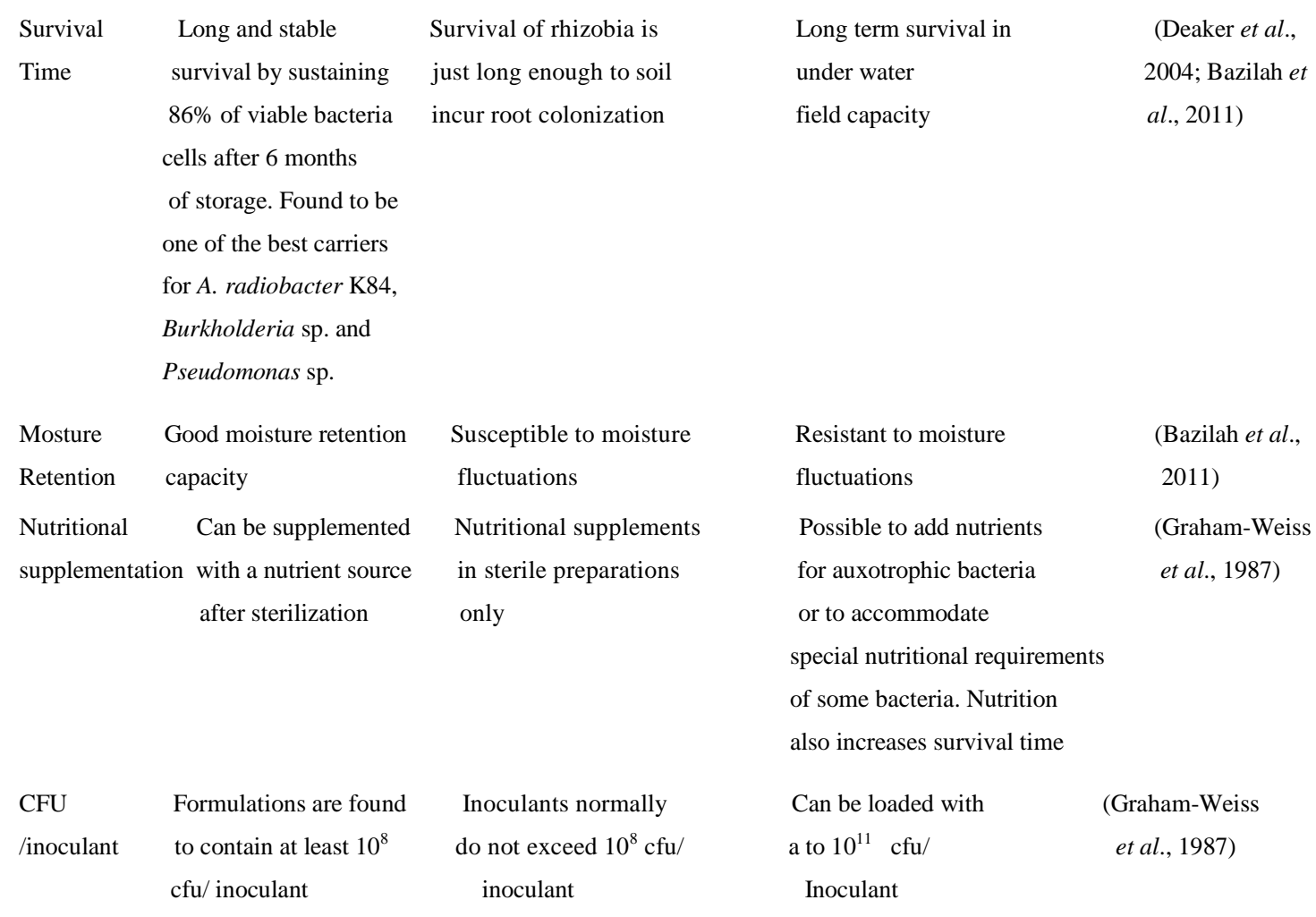

-dried alginate-glycerol beads significantly increased bacterial survival also under UV light radiation (Zohar -Perez et al., 2003). Intraradical structures of $G$. intraradices embedded in alginate beads were still infective after up to 62 months after storage in plastic vials at $4^{\circ} \mathrm{C}$ (Plenchette and Strullu, 2003).

Recently, a process using starch industry wastewater as a carbon source for the production of Sinorhizobium meliloti with simultaneous formulation using alginate and soy oil as emulsifier has been proposed, showing a cell viability of more than $10^{9} \mathrm{CFUmL}^{-1}$ after 9 weeks of storage (Rouissi et al., 2010). Two patents have also been registered: French Patent application no. 77.10254 (Corresponding to U.S. Patent no. 4.155.737) which makes use of a polymer gel based on polyacrylamide gel or a silica gel for different microorganisms; the US patent 5021350 on the process for inclusion of mycorrhizae and actinorhizae in a polymer gel matrix based on at least one polymer from the polysaccharide group, with at least partial crosslinking of the polymer (Malusa et al., 2012)

It appears that alginate is the most promising of the encapsulating materials tested so far but it is still very premature to predict whether it will be able to displace peat in the inoculation technology due to its possible deficiencies, especially their higher price than peat. Although commercial alginate preparations are not yet available for bacterial plant inoculation, several other materials, which are used in industrial and environmental microbiology, may be considered as substitutes when the microorganism fails to adapt to alginate preparations
(Table 3). To the best of our knowledge, almost none have been tested in soil or in the field.

\section{PROMISING NEW TECHNOLOGIES}

One of the methods for storing and delivering microorganisms through liquid formulations is water-in-oil emulsions (Vandergheynst et al., 2006). This formulation slows down water evaporation as the oil traps the water around the organism which is particularly beneficial for organisms that are sensitive to desiccation. Water-in-oil emulsions allow the addition of substances to the oil and/or aqueous phases which could improve both cell viability and release kinetics. However, one of the major issues of concern is cell sedimentation during storage. Thickening the oil phase using hydrophobic silica nanoparticles significantly reduced cell sedimentation and improved cell viability during storage (Vandergheynst et al., 2007).

Recently, a new process named PGSS (Particles from Gas Saturated Solutions), based on the application of supercritical fluid properties is used which is carried out at low temperatures and uses carbon dioxide as a supercritical fluid. The final product of the process is almost spherical particles that form a free-flowing powder which can be suspended in water. The possibilities of the PGSS process have already successfully been demonstrated for several solids and liquids (Cocero et al., 2009).

Another interesting new technology is proposing the exploitation of the natural production of bacterial biofilms as a possible carrier. Two types of biofilms are employed in that case: biofilms growing onto inert 
supports (charcoal, resin, concrete, clay brick, sand particles) in which biofilms grow all around the particles, and the size of the biofilm particles grows with time usually to several $\mathrm{mm}$ in diameter and biofilms that are formed as a result of aggregate formation also called granular biofilm which may take from several weeks to several months (Qureshi et al., 2005). Application of a biofilmed inoculant containing a fungalrhizobia consortium significantly increased $\mathrm{N}_{2}$ fixation in soybean compared to a traditional rhizobium inoculant (Jayasinghearachchi and Seneviratne, 2004). Wheat seedlings inoculated with biofilm-producing bacteria exhibited an increased yield in moderate saline soils (Ashraf et al., 2004). Inocula made with biofilms were shown to allow their rhizobia survive at high salinity (400 $\mathrm{mM} \mathrm{NaCl}$ ) by 105 -fold compared to rhizobial monocultures (Seneviratne et al., 2008). Interestingly, beneficial endophytes were observed to produce higher acidity and plant growth-promoting hormones than their mono- or mixed cultures with no biofilm formation (Bandara et al., 2006).

Bionanotechnology applications which employ nanoparticles made of inorganic or organic materials could also provide new avenues for the development of carrier-based microbial inocula (Malusa et al., 2012). The physical stability and the high surface area of nanotubes, together with the ease and cost-effective fabrication of nanotube membranes may thus expand their use in the production of biofertilizer. The use of nanoformulations may enhance the stability of biofertilizers and biostimulators with respect to dessication, heat, and UV inactivation.

\section{Conclusion}

Microbial inoculants have long been incorporated into field practices worldwide, with satisfactory results, especially for rhizobia. The recent area of interest is the use of plant-growth promoting rhizobacteria (PGPR) as inoculants. The use of PGPRs on wheat and other cereal crops are being taken as an opportunity to be pursued for similar purposes. Yet, there is a little knowledge about the methods which are used for identifying the best bacteria for the task, and even less is known about their rhizocompetence. We need to work on the other characteristics which are required of potentially beneficial bacteria to function, and survive in their new environment. An additional challenge which remains with us is developing improved carriers that can consistently provide higher number of bacteria under field conditions and extend the shelf life of the bacteria.

During the last century, peat formulations have been developed into effective and accepted carriers, but their development has almost reached its limits. Synthetic carriers offer greater potential and flexibility for the inoculation industry but they still have to be transferred from experimental concepts into commercial inoculants. It is quite early to declare these carriers as potentially universal due to the lack of information about new developments from inoculant companies, even though they overcome many of deficiencies of peat-based inoculants. Due to the high cost of development companies are reluctant to develop synthetic inoculants for the target crop, but it might be supported by the bioremediation industry as many types of encapsulated forms of microorganisms has already been developed for bioremedation use.

The demand for natural biofertilizers is rising steadily in all parts of the world. Public awareness about the environment is increasing, while the apprehension about pollution and health hazards due to synthetic chemicals esp. in rich countries is growing (Shukla and Shukla, 2012). Significant advances has been made by some Asian countries in the development and use of biofertilizers but their potential remains largely underutilized due to the difference in efforts and experiences in different countries.

Several policy and technological gaps need to be addressed which includes: Inconsistency in efficacy toxicology and general safety including allergenic risks in inhaling pertinacious materials; the required degree of stringency of regulation; location, characterization and indexing of agents and creation of repositories; characterization of agro-ecological conditions/regions for key traits and raising the thresholds of desired traits; standard and stable products; quality control; matching performance with synthetics; bioprospecting and allied chemical profiling; scientifically sound use packages; well and joint use with the synthetics are some of the technological aspects.

Special attention should be paid to the needs and constraints of developing countries that need easy-to-use and inexpensive formulations as agriculture in developed countries is the major promoter of microbial inoculants that are environmental friendly. For the future, more research should be focused on the development of better and more economical feasible, synthetic inoculant carriers, while sustaining peat-based inoculant production for agriculture. The other options should be considered as long-term goals.

\section{REFERENCES}

Adesemoye, A.O., Obini, M. and Ugoji, E.O. (2008). Comparison of plant growyh promotion with Pseudomonas aerugenosa and Bacillus subtilis in three vegetables. Brazilian J. Microbiol., 39:423-426.

Ahemad, M. and Khan, M.S. (2009). Effect of insecticide -tolerant and plant growth promoting Mesorhizobium on the performance of chickpea grown in insecticide stressed alluvial soils. J Crop Sci. Biotechnol., 12: 213-222.

Ahemad, M. and Khan, M.S.(2010a). Growth promotion and protection of lentil (Lens esculenta) against herbicide stress by Rhizobium species. Ann. Microbiol., 60: 735-745.

Ahemad, M. and Khan, M.S. (2010b). Ameliorative effects of Mesorhizobium sp. MRC4 on chickpea yield and 
yield components under different doses of herbicide stress. Pest. Biochem. Physiol., 98: 183-190.

Ahemad, M. and Khan, M.S.(2010c). Insecticide-tolerant and plant-growth-promoting Rhizobium improves the growth of lentil (Lens esculentus) in insecticide-stressed soils. Pest Manage. Sci., 67: 423-429.

Ahemad, M. and Malik, A. (2011). Bioaccumulation of heavy metals by zinc resistant bacteria isolated from agricultural soils irrigated with wastewater. Bacteriol. J., 2: 12-2.

Ahemad, M. (2012). Implications of bacterial resistance against heavy metals in bioremediation: a review. IIOABJ., 3: 39-46.

Ahemad, M. and Khan, M.S. (2012). Effects of pesticides on plant growth promoting traits of Mesorhizobium strain MRC4 J. Saudi Soc. Agric. Sci., 11: 63-71.

Ahemad, M. and Kibret, M. (2014). Mechanisms and applications of plant growth promoting rhizobacteria: Current perspective. J. king Saud Univ Sci., 26(1): 1-20.

Ajay, K., Rawlat, A.K., Verman, L.N., Khare, A.K. and Kaushal, A. (1996). Oxalic acid industrial waste as a carrier for Rhizobium inoculants and its effect on soybean. J. Indian Society Soil Sci., 44: 249-252.

Alexandre-Franco, M., Albarran-Liso, A. and Gomez-Serrano, V. (2011). An identification study of vermiculites and micas: Adsorption of metal ions in aqueous solution. Fuel Processing Tech., 92(2): 200-205.

Amer, G.A. and Utkhede, R.S. (2000). Development of formulations of biological agents for management of root rot of lettuce and cucumber. Canadian J. Microbiol., 46: 809-816.

Anandaraj, B. and Delapierre, A.L.R. (2010). Studies in influence of bioinoculants (Pseudomonas fluorescens, Rhizobium sp., Bacillus megaterium) in green gram. $J$. Biosci. Tech., 1(2): 95-99.

Anjum, M.A., Sajjad, M.R., Akhtar, N., Qureshi, M., Iqbal, A., Rehman, J.A. and Mahmud-ul-Hasan (2007). Response of cotton to plant growth promoting rhizobacteria (PGPR) inoculation under different levels of nitrogen. $J$. Agric. Res., 45: 135-143.

Antoun, H. and Pre'vost, D. (2005). Ecology of plant growth promoting rhizobacteria. In: Siddiqui, Z.A. (ed) PGPR: biocontrol and biofertilization. Dordrecht., 1-38.

Ashraf, M., Hasnain, S., Berge, O. and Mahmood, T. (2004). Inoculating wheat seedlings with exopolysaccharide -producing bacteria restricts sodium uptake and stimulates plant growth under salt stress. J. Food Sci., 71(3): 89-99.

Badawi, F.S.F., Biomy, A.M.M. and Desoky, A.H. (2011). Peanut plant growth and yield as influenced by co-inoculation with Bradyrhizobium and some rhizo-microorganisms under sandy loam soil conditions. Annls. Agric. Sci., 56(1):17-25.

Bai, Y.M., Zhou, X. and Smith, D.L. (2003). Enhanced soybean plant growth resulting from coinoculation of Bacillus strains with Bradyrhizobium japonicum. Crop Sci., 43:1774-1781.

Bandara, W.M.M.S.; Seneviratne, G. and Kulasooriya, S.A. (2006). Interactions among endophytic bacteria and fungi: effects and potentials. J Bioscience, 31(5): 645-650.

Bargali, K. (2011). Screening of leguminous plants for VAM association and their role in restoration of degraded lands. J. American Sci., 7(1): 7-11.

Bashan, Y. (1986). Alginate beads as synthetic inoculant carriers for the slow release of bacteria that affect plant growth. Appl. Environ. Microbiol. 51:1089-1098.

Bashan, Y., Levanony, H. and Ziv-Vecht, O. (1987). The fate of field-inoculated Azospirillum brasilense $\mathrm{Cd}$ in wheat rhizosphere during the growing season. Can. J. Microbiol., 33:1074-1079.

Bashan, Y., Holguin, G. and Puente, M.E. (1992). Alternativa agricola regional por fertilizantes bacterianos. In: Ortega, A. (ed) Uso y Manejo de los Recursos Naturales en la Sierra de la Laguna Baja California Sur, CIB Press, La Paz, Mexico. (in Spanish), pp 47-67.

Bashan, Y. and Holguin, G. (1997). Azospirillum-plant relationships: Environmental and physiological advances (1990-1996). Can. J. Microbiol., 43: 103-121.

Bashan, Y. (1998). Inoculants of plant growth-promoting bacteria for use in agriculture. Biotech. Adv., 16:729-770.

Bashan, Y., Luis, A.J.P.H. and Bacilio, L.M. (2002). Alginate microbeads as inoculant carriers for plant growth -promoting bacteria. Biol. Fertil. Soils, 35: 359-368.

Bazilah, A.B.I., Sariah, M., Abidin, M.A.Z. and Yasmeen, S. (2011). Effect of carrier and temperature on the viability of Burkholderia sp. (UPMB3) and Pseudomonas sp. (UPMP3) during Storage. Int. J. Agri. Biol., 13:198-202.

Beneduzi, A., Peres, D., Vargas, L.K., Bodanese-Zanettini, M.H. and Passaglia L.M.P. (2008). Evaluation of genetic diversity and plant growth promoting activities of nitrogen-fixing Bacilli isolated from rice fields in South Brazil. Appl. Soil Ecol., 39: 311-320.

Bhattacharyya, P.N. and Jha D.K. (2012). Plant growth -promoting rhizobacteria (PGPR): emergence in agriculture. World J. Microbiol. Biotechnol., 28: 1327-1350.

Biswas, B. and Gresshoff, P.M. (2014). The role of symbiotic nitrogen fixation in sustainable production of biofuels. Int. J. Mol. Sci., 15: 7380-7397.

Bissonnette, N., Lalande, R. and Bordeleau, L.M. (1986). Large-scale production of Rhizobium meliloti on whey. Appl. Env. Microbiol., 52: 838-841.

Bissonnette, N. and Lalande, R. (1988). High survivability of cheese whey-grown Rhizobium meliloti cells upon exposure to physical stress. Appl. Env. Microbiol., 54: 183-187.

Black, M., Moolhuijzen, P., Chapman, B.; Barrero, R.; Howieson, J., Hungria, M. and Bellgard, M. (2012). The genetics of symbiotic nitrogen fixation: comparative genomics of 14 Rhizobia strains by resolution of protein clusters. Genes, 3: 138-166.

Bora, T., Ozaktan, H., Gore. E and Aslan, E. (2004). Biological control of Fusarium oxysporum f. sp. melonis by wettable powder formulations of the two strains of Pseudomonas putida. J. Phytopathol., 152: 471-475.

Bozzolo, A. and Evans, M.R. (2013). Efficacy of cork granulates as a top coat substrate component for seed germination as compared to vermiculite. Horttechnol., 23: 114-118.

Braud, A., Jézéquel, K., Bazot, S. and Lebeau, T. (2009). Enhanced phytoextraction of an agricultural $\mathrm{Cr}-, \mathrm{Hg}$ and $\mathrm{Pb}$-contaminated soil by bioaugmentation with siderophore producing bacteria Chemosphere, 74: 280-286.

Brockwell, J. (1985). Environmental interactions influencing innovative practices in legume inoculation, In: Shibles R (ed) Proceedings of the World Soybean Conference III, Westview Press, Boulder, Colo, pp. 943-950.

Bustamante, M.A., Paredes, C., Moral, R.; Agullo, E., Perez -Murcia, M.D. and Abad, M. (2008). Composts from distillery wastes as peat substitutes for transplant production. Resour. Conserv. Recycl. 52:792-799. 
Cakmakci, R., Erat, M., Erdogan, U.G. and Donmez, M.F. (2007). The influence of PGPR on growth parameters, anti-oxidant and pentose phosphate oxidative cycle enzymes in wheat and spinach plants. J. Plant Nutr. Soil Sci., 170: 280-295.

Caesar, A.J. and Burr, T.J. (1991). Effect of conditioning, betaine, and sucrose on survival of rhizobacteria in powder formulations. Appl. Environ. Microbiol., 57: $168-172$.

Cassidy, M.B., Lee, H. and Trevors, J.T. (1997). Survival and activity of lac-lux marked Pseudomonas aeruginosa UG2Lr cells in encapsulated kcarageenan over 4 years at $48^{0}$ C. J. Microbiol. Meth., 30: 167-170.

Chao, W.L. and Alexander, M. (1984). Mineral soils as carrier for Rhizobium inoculants. Appl. Environ. Microbiol., 47: 94-97.

Cocero, M.J., Martin, A., Mattea, F. and Varona, S. (2009). Encapsulation and co-precipitation processes with supercritical fluids: fundamentals and applications. $J$. Supercrit Fluid, 47(3): 546-555.

Crawford, S.L. and Berryhill, D.L. (1983). Survival of Rhizobium phaseoli in coal-based legume inoculants applied to seeds. Appl. Environ. Microbiol., 45:703-705.

Dardanelli, M.S., Fernández, F.J., Espuny, M.R., Rodrı'guez, M.A., Soria, M.E., Gil Serrano, A.M., Okon, Y. and Megi'as, M. (2008). Effect of Azospirillum brasilense coinoculated with Rhizobium on Phaseolus vulgaris flavonoids and Nod factor production under salt stress. Soil Biol. Biochem., 40: 2713-2721.

Dary, M., Chamber-Pérez, M.A., Palomares, A.J. and Pajuelo, E. (2010). In situ" phytostabilisation of heavy metal polluted soils using Lupinus luteus inoculated with metal resistant plant-growth promoting rhizobacteria. $J$. Hazard. Mater., 177: 323-330.

Daza, A., Santamaria, C., Rodriguez-Navarro, D.N., Camacho, M., Orive, R. and Temprano, F. (2000). Perlite as a carrier for bacterial inoculants. Soil Biol. Biochem., 32: 567-572.

Deaker, R., Roughley, R. and Kennedy, I.R. (2004). Legume seed inoculation technology - a review. Soil Biol. Biochem., 36: 1275-1288.

Dell'Amico, Cavalca, L. and Andreoni,V. (2008). Improvement of Brassica napus growth under cadmium stress by cadmium resistant rhizobacteria. Soil Biol. Biochem., 40: 74-84.

Döbereiner, J. and Day, J.M. (1976). Associative symbioses in tropical grasses: characterization of microorganisms and dinitrogen-fixing sites. In: Newton,W.E., Nyman, C.J. (ed) Proceedings of the first International Symposium on Nitrogen Fixation. Vol. 2, Washington State University Press, Pullman, USA. pp 518-538.

Egamberdiyeva, D. (2007). The growth and nutrient uptake of maize inoculated with plant growth promoting bacteria affected by different soil types. Appl. Soil Ecol., 36: 184 $-189$.

Einarsson, S., Gudmundsson, J., Sverrisson, H., Kristjansson, J.K. and Runolfsson, S. (1993). Production of Rhizobium inoculants for Lupinus nootkatensis on nutrient -supplemented pumice. Appl. Environ. Microbiol., 59: 3666-3668.

Fages, J. (1992). An industrial view of Azospirillum inoculants: formulation and application technology. Symbiosis, 13: 15-26.

Faisal, M. and Hasnain, S. (2005). Bacterial Cr (VI) reduction concurrently improves sunflower (Helianthus annuus L.) growth. Biotechnol. Lett., 27: 943-947.
Felici, C., Vettori, L., Giraldi, E., Forino, L.M.C., Toffanin, A., Tagliasacchi, A.M. and Macro Nuti M. (2008). Single and coinoculation of Bacillus substilis and Azospirillum brasilense on Lycoperscion esculentum: Effects on plant growth and rhizoaphere microbial community. Appl. Soil Ecol., 10: 260-270.

Ghevariya, K.K. and Desai, P.B. (2014) Rhizobacteria of sugarcane: In vitro screening for their plant Growth Promoting potentials. Res. J. Recent. Sci., 3: 52-58.

Gholami, A., Shahsavani, S. and Nezarat S. (2009). The effect of plant growth promoting rhizobacteria (PGPR) on germination, seedling growth and yield of maize. Int. J. Biol. Life Sci., 1: 35-40.

Gil, M.V., Calvo, L.F., Blanco, D. and Sanchez, M.E. (2008). Assessing the agronomic and environmental effects of the application of cattle manure compost on soil by multivariate methods. Bioresour. Technol., 99:5763-5672.

Glick, B.R. and Bashan, Y. (1997). Genetic manipulation of plant growth-promoting bacteria to enhance biocontrol of phytopathogens. Biotechnol. Adv., 15:353-378.

Glick, B.R. (2012). Plant Growth-Promoting Bacteria: Mechanisms and Applications. Hindawi Publishing Corporation, Scientifica.

Graham-Weiss, L.; Bennett, M.L. and Alan, S.P. (1987). Production of bacterial inoculants by direct fermentation on nutrient-supplemented Vermiculite. Appl. Environ. Microbiol., 53(9): 2138-2140.

Gunasekaran, S., Balachandar, D. and Mohanasundaram, K. (2004). Studies on synergism between Rhizobium, plant growth promoting rhizobacteria (PGPR) and phosphate solubilizing bacteria in blackgram. In: Kannaiyan, S.; Kumar, K.; Govimdarajan, K. (ed) Biofertilizer technology for rice based cropping system, Scientific Publ. Jodhpur, pp. 269-273.

Hartley, E.J., Gemmell, L.G., Slattery, J.F., Howieson, J.G. and Herridge, D.F. (2005). Age of peat-based lupin and chickpea inoculants in relating to quality and efficacy. Australian J. Exp. Agr., 45: 183-188.

Hay, I.D., Rehman, Z.U., Ghafoor, A. and Rehm, B.H.A. (2010). Bacterial biosynthesis of alginates. J. Chem. Technol. Biotechnol., 85: 752-759.

Hemphill, D.D.Jr. (1982). Anticrustant effects on soil mechanical resistance and seedling emergence. Hort. Sci., 17: 391-393.

Hernandez, A., Weekers, F., Mena, J., Borroto, C. and Thonart, P. (2006). Freeze-drying of the biocontrol agent Tsukamurlla paurometabola, C-924: predicted stability of formulated powders. Ind. Biotechnol., 2(3): 209-212.

Jahanian, A., Chaichi, M.R., Rezaei, K., Rezayazdi, K. and Khavazi K. (2012). The effect of plant growth promoting rhizobacteria (pgpr) on germination and primary growth of artichoke (Cynara scolymus). Int. J. Agric. Crop Sci., 4: 923-929.

Jain R, Saxena J, Sharma V. (2010). The evaluation of free and encapsulated Aspergillus awamori for phosphate solubilization in fermentation and soil-plant system. Applied Soil Ecol.; 46: 90-94.

Jayasinghearachchi, H.S. and Seneviratne, G. (2004). A bradyrhizobial-Penicillium spp. biofilm with nitrogenise activity improves $\mathrm{N}_{2}$ fixing symbiosis of soybean. Biol. Fert. Soils, 40(6): 432-434.

Joe, M.M., Saravanan, V.S., Islam, M.R and Sa T. (2014). Development of alginate-based aaggregate of 
Methylobacterium sp. and Azospirillum brasilense tested under in vitro conditions to promote plant growth. J. Appl. Microbiol., 116(2)

Jones, K.A. and Burges, H.D. (1998). Technology of

formulation and application. In: Burges, H.D. (ed) Formulation of microbial pesticides: beneficial microorganisms, nematodes and seed treatments. Kluwer Academic Publishers, Dordrecht, pp 7-29.

Joshi, N.V. (1920). Studies on the root nodule organism of the leguminous plant. India Dept. Agr. Mem., Bact. Ser., 1, 247-276.

Kaljeet, S., Keyeo, F. and Amir, H.G. (2011). Influence of carrier materials and storage temperature on survivability of rhizobial inoculant. Asian J. Plant Sci., 10: 331-337.

Kalra, A., Chandra, M., Awasthi, A., Singh, A.K. and Khanuja, S.P.S. (2010). Natural compounds enhancing growth and survival of rhizobial inoculants in vermicompost -based formulations. Biol. Fertil. Soils., 46: 521-524.

Keppeler, S., Ellis, A. and Jacquier, J.C. (2009). Cross-linked carrageenan beads for controlled release delivery systems. Carbohydr. Polym., 78: 973-977.

Keyser, H.H., Somasegaran, P. and Bohlool, B.B. (1993). Rhizobial ecology and technology. In: Metting, E.B., editor. Soil Microbial Ecology: Applications in Agricultural and Environmental Management. New York, NY, USA: Marcel Dekker;. pp. 205-226.

Khan, M.S., Zaidi, A., Wani, P.A. and Oves, M. (2009). Role of plant growth promoting rhizobacteria in the remediation of metal contaminated soils. Environ. Chem. Lett., 7: 1-19.

Klein, J., Stock, J. and Vorlop, K.D. (1983). Pore size and properties of spherical Ca-alginate biocatalysts. Appl. Microbiol. Biotechnol., 18(2): 86-91.

Kloepper, J.W. and Schroth, M.N. (1981). Development of powder formulation of rhizobacteria for inoculation of potato seed pieces. Phytopathol., 71: 590-592.

Kloepper, J.W., Zablotowick, R.M., Tipping, E.M. and Lifshitz, R. (1991). Plant growth promotion mediated by bacterial rhizosphere colonizers. In: Keister, D.L.; Cregan, P.B. (Eds.), The Rhizosphere and Plant Growth. Kluwer Academic Publishers, Dordrecht, Netherlands, pp. 315-326.

Ko, H.J., Kim, K.Y., Kim, H.T., Kim, C.N. and Umeda, M. (2008). Evaluation of maturity parameters and heavy metal contents in composts made from animal manure. Waste Manage., 28: 813-820.

Kohler, J., Caravaca, F. and Roldan, A. (2010). An AM fungus and a PGPR intensify the adverse effects of salinity on the stability of rhizosphere soil aggregates of Lactuca sativa. Soil Biol. Biochem., 42(3): 429-434.

Kostov, O. and Lynch, J.M. (1998). Composted sawdust as a carrier for Bradyrhizobium, Rhizobium and Azospirillum in crop inoculation. World J. Microbiol. Biotechnol., 14: 389-397.

Kremer, R.J. and Peterson, H.L. (1983). Field evaluation of selected rhizobium in an improved legume inoculant. Agron. J., 75: 139-143.

Kumar, A., Kumar, A., Devi, S., Patil, S., Payal, C. and Negi, S. (2012). Isolation, screening and characterization of bacteria from rhizospheric soils for different plant growth promotion (PGP) activities: an in vitro study. Recent Res. Sci. Technol., 4(1): 01-05.

Ladha, J.K. and Reddy, P.M. (1995). Extension of nitrogen fixation to rice- Necessity and possibilities. Geojournal, 35(3): 363-372.

Lima, J.deA., Sonza, A.F., Castor, O.S. and de Menezes
-Sobrinho, J.A. (1984). Effects of organic matter and vermiculite on garlic yields. Pesqui. Agropecu. Bras., 19: 41-45.

Ma, Y., Rajkumar, M. and Freitas, H. (2009a). Isolation and characterization of Ni mobilizing PGPB from serpentine soils and their potential in promoting plant growth and $\mathrm{Ni}$ accumulation by Brassica spp. Chemosphere, 75(6): 719-725.

Ma, Y., Rajkumar, M. and Freitas, H. (2009b). Improvement of plant growth and nickel uptake by nickel resistant -plant-growth promoting bacteria. J. Hazard. Mater., 166: 1154-1161.

Ma, Y., Rajkumar, M., Luo, Y. and Freitas, H. (2011). Inoculation of endophytic bacteria on host and non-host plants-effects on plant growth and Ni uptake. J. Hazard. Mater., 195: 230-237.

Maheshwari, D.K., Kumar, S., Kumar, B. and Pandey, P. (2010). Co-inoculation of urea and DAP tolerant Sinorhizobium meliloti and Pseudomonas aeruginosa as integrated approach for growth enhancement of Brassica juncea. Indian J Microbiol., 50(4): 425-431.

Malusa, E., Sas-Paszt, L. and Ciesielska, J. (2012). Technologies for beneficial microorganisms inocula used as biofertilizers. T. Sentific World J., Article ID 491206, pp 12.

Manikandan, R., Saravanakumar, D., Rajendran, L., Raguchander, T. and Samiyappan, R. (2010). Standardization of liquid formulation of Pseudomonas fluorescens $\mathrm{Pf} 1$ for its efficacy against Fusarium wilt of tomato. Biol. Control, 54: 83-89.

McInnes, A. and Date, R.A. (1999). Improving survival of rhizobia on Stylosanthes and Desmanthus seed at high temperature. Proceedings of the $12^{\text {th }}$ Australian Nitrogen Fixation Conference, Country Comfort Hotel, Wagga Wagga, Australia, pp 3-4.

Meisinger, A.C. (1984). Vermiculite, In Bureau of Mines minerals yearbook, vol. 1. Superintendent of Documents, Government Printing Office, Washington, D.C. p 1-4.

Mia, M.A.B. and Shamsuddin, Z.H. (2010). Rhizobium as a crop enhancer and biofertilizer for increased cereal production. African J Biotechnol., 9(37): 6001-6009.

Mishra, P.K., Bisht, S.C., Ruwari, P., Joshi, G.K., Singh, G., Bisht, J.K. and Bhatt, J.C. (2011). Bioassociative effect of cold tolerant Pseudomonas spp. and Rhizobium leguminosarum-PR1 on iron acquisition, nutrient uptake and growth of lentil (Lens culinaris L.). European J. Soil Biol., 47(1): 35-43.

Moral, R., Paredes, C., Bustamante, M.A., Egea, F.M. and Bernal, M.P. (2009). Utilization of manure composts by high value crops: Safety and environmental challenges. Biroresour. Technol., 100(22): 5454-5460.

Mugnier, J. and Jung, G. (1985). Survival of bacteria and fungi in relation to water activity and the solvent properties of water in biopolymer gels. Appl. Environ. Microbiol., 50: 108-114.

Muniruzzaman, S. and Khan, S.I. (1992). Suitability of some local agro-industrial wastes as carrier materials for Rhizobium. sp. infecting Sesbania bispinosa. World J. Microbiol. Biotechnol., 8: 329-330.

Muresu, R., Sulas, L. and Caredda, S. (2003). Legume Rhizobium symbiosis: characteristics and prospects of inoculation. Rivoluzione Agronomica, 37: 33-45.

Muthukumarasamy, R., Revathi, G. and Lakshminarasimhan, C. (1999). Diazotrophic associations in sugarcane cultivation in South India. Trop. Agric., 76: 171-178. 
Neyra, C.A., Atkinson, A. and Olubayi, O. (1995). Coaggregation of Azospirillum with other, bacteria: basis for functional diversity. In: Fendrik, I.; Gallo, M.D.; Vanderleyden, J.; de Zamaroczy, M. (ed) Azospirillum VI and related microorganisms, genetics-physiology-ecology, Vol. G37:429-439, NATO ASI Series, Series G: Ecological Sciences, Springer Verlag, Berlin, Heidelberg, Germany.

Nobbe, F. and Hiltner, L. (1896). U.S. Patent 570813. Inoculation of the soil for cultivating leguminous plants.

Orlando, P., Binaglia, L., De Feo, A., Trevisi, R., Melodia, C. and Trenta, R. (1994). Preparation of high molecular weight radioiodinated alginic acid. J. Labelled Compd. Radiopharm, 34: 653-657, doi: 10.1002/jlcr. 2580340709.

Pandey, R. and Khuller, G.K. (2005). Alginate as a drug delivery carrier- handbook of carbohydrate engineering. Taylor \& Francis Group, LLC, pp 799-815.

Park, J.K. and Chang, H.N. (2000). Microencapsulation of microbial cells. Biotechnol. Adv., 18: 303-319.

Partha, N. and Sivasubramanian, V. (2006). Recovery of chemicals from pressmud -a sugar industry waste. Indian Chemical Engr., 48(3): 161-163.

Plenchette, C. and Strullu, D.G. (2003). Long-term viability and infectivity of intraradical forms of Glomus intraradices vesicles encapsulated in alginate beads. Mycological Res., 107(5): 614-616.

Pooet, D.T., Dhulster, P., Barbotin, J.N. and Thomas, D. (1986). Plasmid inheritability and biomass production: comparison between free and immobilized cell cultures of Eschrichia coli BZ18 (pTG201) without selection pressure. J. Bacteriol., 165: 871-877.

Qureshi, N., Annous, B.A., Ezeji, T.C., Karcher, P. and Maddox, I.S. (2005). Biofilm reactors for industrial bioconversion process: employing potential of enhanced reaction rates. Microb Cell Factories, 4, article 24.

Rajendran, G., Sing, F., Desai, A.J. and Archana G. (2008). Enhanced growth and nodulation of Pigeon Pea by co-inoculation of Bacillus strains with Rhizobium spp. Biosource Technol. 99(11): 4544-4550.

Rajkumar, M., Nagendran, R., Kui, J.L., Wang, H.L. and Sung Z.K. (2006). Influence of plant growth promoting bacteria and $\mathrm{Cr}$ (VI) on the growth of Indian mustard. Chemosphere, 62: 741-748.

Rani, A., Souche Y.S. and Goel R. (2009). Comparative assessment of in situ bioremediation potential of cadmium resistant acidophilic Pseudomonas putida 62BN and alkalophilic Pseudomonas monteilli 97AN strains on soybean. Int. Biodeterior. Biodegrad., 63: 62-66.

Rassis, D., Nussinovitch, A. and Saguy, I.S. (2002). Collapse, shrinkage and structural changes in dried alginate gels containing fillers. Food Hydrocolloid, 16(2): 139-151.

Rebah, F.B., Tyagi, R.D. and Prevost, D. (2001). Acid and alkaline treatments for enhancing the growth of rhizobia in sludge. Canadian J. Microbiol., 47: 467-474.

Rebah, F.B., Tyagi, R.D., Prevost, D. and Surampalli, R.Y. (2002a). Wastewater sludge as a new medium for rhizobial growth. Water Qual. Res. J. Canada, 37: 353-370.

Rebah, F.B., Tyagi, R.D. and Prevost, D. (2002b). Nodulation and yield of alfalfa grown in sludge amended soils and inoculated with rhizobia produced in sludge. J. Environ. Qual., 31: 1339-1348.

Rebah, F.B.; Prevost, D.; Yezza, A. and Tyagi, R.D. (2007). Agro-industrial waste materials and wastewater sludge for rhizobial inoculant production: A review. Bioresource Technol., 98(18): 3535-3546.
Reid, W.S., Liptay, A., Nicholls, C.F. and Marriage, P.B. (1983). A plug-mix planter attachment for dispensing a charcoal vermiculite mixture to protect emerging seedlings from herbicide toxicity. Canadian J. Plant Sci., 63: 567-571.

Rekha, P.D., Lai, W., Arun, A.B. and Young C. (2007). Effect of free and encapsulated Pseudomonas putida CC-R2-4 and Bacillus subtilis CC-pg104 on plant growth under gnotobiotic conditions. Biores. Technol., 98: 447-451.

Rouissi, T., John, R.P., Brar, S.K., Tyagi, R.D. and Prevost, D. (2010). Original research: centrifugal recovery of rhizobial cells from fermented starch industry wastewater \& development of stable formulation. Ind Biotechnol., 6 (1): 41-49.

Saharan, K., Sarma, M.V.R.K., Srivastava, R., Sharma, K., Johri, B.N., Prakash, A., Sahai, V. and Bisaria, V.S. (2010). Development of non-sterile inorganic carrier -based formulations of fluorescent pseudomonad R62 and R81 and evaluation of their efficacy on agricultural crops. Appl. Soil Ecol., 46: 251-258.

Saharan, B.S. and Nehra, V. (2011). Plant Growth Promoting Rhizobacteria: A Critical Review. Life Sci. Med. Res., 21. http://astonjournals.com/lsmr.

Sahin, F., Cakmakci, R. and Kantar, F. (2004). Sugar beet and barley yields in relation to inoculation with $\mathrm{N}_{2}$-fixing and phosphate solubilizing bacteria. Plant Soil, 265: 123-129.

Sangeetha, D. and Stella D. (2012). Survival of plant growth promoting bacterial inoculants in different carrier materials. Int. J. Pharm. Biol. Arch., 3(1): 170-178.

Saravanakumar, D., Harish, S., Loganathan, M., Vivekananthan, R., Rajendran, L. and Samiyappan, R. (2007a). Rhizobacterial bioformulation for the effective management of Macrophomina root rot in mungbean. Arch. Phytopathol. Plant Protect., 40(5): 323-337.

Saravanakumar, D., Vijayakumar, C., Kumar, N. and Samiyappan, R. (2007b). PGPR induced defense responses in tea plants against blister blight disease. Crop Protect., 26: 556-565.

Seneviratne, G., Zavahir, J.S., Bandara, W.M.M.S. and Weerasekara, M.L.M.A.W. (2008). Fungal-bacterial biofilms: their development for novel biotechnological applications. World J. Microbiol. Biotechnol., 24(6): 739-743.

Sheng, X.F. and Xia J.J. (2006). Improvement of rape (Brassica napus) plant growth and cadmium uptake by Cadmium resistant bacteria. Chemophore, 64: 1036-1042.

Shukla, R. and Shukla, A. (2012). Market potential for biopesticides: a green product for agricultural application. Int. J. Manag. Res. Rev., 2(1): 91-99.

Siddiqui, Z.A. and Kataoka, R. (2011). Mycorrhizal Inoculants: Progress in Inoculant Production Technology. In: Ahmad, et al. (ed) Microbes and Microbial Technology., Agricultural and Environmental applications 506, DOI: 10.1007/978-1-4419-7931-5-18.

Smith, R.S. (1992). Legume inoculant formulation and application. Canadian J. Microbiol., 38: 485-492.

Smit, E., Wolters, A.C., Lee, H., Trevors, J.T. and van Elsas, J.D. (1996). Interaction between a genetically marked Pseudomonas fluorescens strain and bacteriophage $\varnothing \mathrm{R} 2 \mathrm{f}$ in soil: Effects of nutrients, alginate encapsulation, and the wheat rhizosphere. Microb. Ecol., 31: 125-140.

Sparrow, S.D. and Ham, G.E. (1983a). Nodulation, $\mathrm{N}_{2}$ fixation, and seed yield of navy beans as influenced by inoculant 
rate and inoculant carrier. Agron. J., 75: 20-24.

Sparrow, S.D. and Ham, G.E. (1983b). Survival of Rhizobium phaseoli in six carrier materials. Agron. J., 75: 181-184.

Stajkovic, O., Delic, D., Josic, D., Kuzmanovic, D., Rasulic, N. and Knezevic-Vukcevic, J. (2011). Improvement of common bean growth by co-inoculation with Rhizobium and plant growth-promoting bacteria. Romanian Biotechnol. Lett., 16(1): 5919-5926.

Stephens, J.H.G. and Rask, H.M. (2000). Inoculant production and formulation. Field Crops. Res., 65: 249-258.

Strijdom, B.W. and van Rensburg, H.J. (1981). Effect of steam sterilization and gamma irradiation of peat on quality of Rhizobium inoculants. Appl. Environ. Microbiol., 41: 1344-1347.

Strullu, D-G. and Plenchette, C. (1991). The entrapment of Glomus sp. in alginate beads and their use as root inoculum. Mycological Res., 95: 1194-1196.

Tal, Y., van Rijn, J. and Nussinovitch, A. (1997). Improvement of structural and mechanical properties of denitrifying alginate beads by freeze-drying. Biotechnol. Progr., 13 (6): 788-793.

Tal, Y., van Rijn, J. and Nussinovitch, A. (1999). Improvement of mechanical and biological properties of freeze-dried denitrifying alginate beads by using starch as a filler and carbon source. Appl. Microbiol. Biotechnol., 51(6): 773-779.

Tang, W.H. and Yang, H. (1997). Research and application of biocontrol of plant diseases and PGPR in China. In: Ogoshi, A.; Kobayashi, K.; Homma, Y.; Kodama, F.; Kondo, N.; Akino, S. (ed) Plant Growth-Promoting Rhizobacteria -present status and future prospects, Faculty of Agriculture, Hokkaido University, Sapporo, Japan. pp 4-9.

Tchebotar, V.K., Kang, U.G., Asis, C.A. Jr. and Akao, S. (1998). The use of GUS-reporter gene to study the effect of Azospirillum-Rhizobium coinoculation on nodulation of white clover. Biol. Fertil. Soils, 27: 349-352.

Thakuria, D., Talukdar, N.C., Goswami, C., Hazarika, S., Boro, R.C. and Khan M.R. (2004). Characterization and screening of bacteria from rhizosphere of rice grown in acidic soils of Assam. Curr. Sci., 86: 978-985.

Tittabutr, P., Payakapong, W., Teaumroong, N., Singleton, P.W. and Boonkerd, N. (2007). Growth, survival and field performance of Bradyrhizobial liquid inoculant formulations with Polymeric additives. Sci. Asia, 33: 69-77.

Trevors, J.T., van Elsas, J.D., Lee, H. and van Overbeek, L.S. (1992). Use of alginate and other carriers for encapsulation of microbial cells for use in soil. Microb. Releases, 1: 61-69.

Trevors, J.T., van Elsas, J.D., Lee, H. and Wolters, A.C. (1993). Survival of alginate encapsulated Pseudomonas fluorescens cells in soil. Appl. Microbiol. Biotechnol., 39: 637-643.

Vandergheynst, J.S., Scher, H. and Hong-Yun, G. (2006). Design of formulations for improved biological control agent viability and sequestration during storage. Ind Biotechnol., 2(3): 213-219.

Vandergheynst, J.S., Scher, H.B., Guo, H.Y. and Schultz, D.L. (2007). Water-in-oil emulsions that improve the storage and delivery of the biolarvacide Lagenidium giganteum. BioControl, 52(2): 207-229.

Van Elsas, J.D. and Heijnen, C.E. (1990). Methods for the introduction of bacteria in soil: a review. Biol. Fertil.
Soils, 10: 127-133.

van Veen, J.A., van Overbeek, L.S. and van Elsas, J.D. (1997). Fate and activity of microorganisms introduced into soil. Microbiol. Mol. Biol. Rev., 61(2): 121-135.

Vassileva, M., Serrano, M., Bravo, V., Jurado, E., Nikolaeva, I., Martos, V. and Vassilev, N. (2010). Multifunctional properties of phosphate-solubilizing microorganisms grown on agro-industrial wastes in fermentation and soil conditions. Appl. Microbiol. Biotechnol., 85 (5):1287-1299.

Vassilev, N., Nikolaeva, I. and Vassileva, M. (2005). Polymer -based preparation of soil inoculants: applications to arbuscular mycorrhizal fungi. Rev. Environ. Sci. Biotechnol., 4(4): 235-243.

Vessey, J.K. (2003). Plant growth promoting bacteria as Biofertilisers. Plant Soil., 255: 571-586.

Vidhyasekaran, P. and Muthamilan, M. (1995). Development of formulations of Pseudomonas fluorescens for control of chickpea wilt. Plant Dis., 79:782-786.

Weir, S.C., Dupuis, S.P., Providenti, M.A., Lee, H. and Trevors, J.T. (1995). Nutrient enhanced survival of and phenanthrene mineralization by alginate encapsulated and free Pseudomonas sp. UG14Lr cells in creosotecontaminated soil slurries. Appl. Microbiol. Biotechnol., 43: 946-951.

Weiss, L.G., Bennett, M.L. and Paau, A.S. (1987). Production of bacterial inoculants by direct fermentation on nutrient -supplemented vermiculite. Appl. Environ. Microbiol., 53: 2138-2140.

Xavier, I.J., Holloway, G. and Leggett, M. (2004). Development of rhizobial inoculant formulations. Online. Crop Manage., doi:10.1094/CM-2004-0301-06-RV.

Yabur, R., Bashan, Y. and Hernández-Carmona, G. (2007). Alginate from the macroalgae Sargassum sinicola as a novel source for microbial immobilization material in wastewater treatment and plant growth promotion. $J$. Appl. Phycol., 19(1): 43-53.

Young, C.C., Rekha, P.D., Lai, W.A. and Arun, A.B. (2006). Encapsulation of plant growth-promoting bacteria in alginate beads enriched with humic acid. Biotechnol. Bioengg., 95(1):77-83.

Zahir, Z.A., Arshad, M. and Frankenberger, W.T. (2004). Plant growth promoting rhizobacteria: Applications and perspectives in agriculture. Adv. Agron., 81: 97-168.

Zaidi, A., Khan, M.S., Ahemad M. and Oves, M. (2009). Plant growth promotion by phosphate solubilizing bacteria. Acta Microbiol. Immunol. Hungarica, 56: 263-284.

Zhao, H., Li, M., Fang, K., Chen, W. and Wang, J. (2012). In: Silico Insights into the Symbiotic Nitrogen Fixation in Sinorhizobium melilotivia Metabolic Reconstruction. PLOS ONE, 7(2): e31287.

Zohar-Perez, C., Ritte, E., Chernin, L., Chet, I. and Nussinovitch, A. (2002). Preservation of chitinolytic Pantoae agglomerans in a viable form by cellular dried alginate-based carriers. Biotechnol. Prog., 18: 1133-1140.

Zohar-Perez, C., Chernin, L., Chet, I. and Nussinovitch, A. (2003). Structure of dried cellular alginate matrix containing fillers provides extra protection for microorganisms against UVC radiation. Radiat Res., 160(2): 198-204.

Zohar-Perez, C., Chet, I. and Nussinovitch, A. (2005). Mutual relationships between soils and biological carrier systems. Biotechnol. Bioeng., 92(1): 54-60. 